ARTICLE

\title{
PTEN $\alpha$ functions as an immune suppressor and promotes immune resistance in PTEN-mutant cancer
}

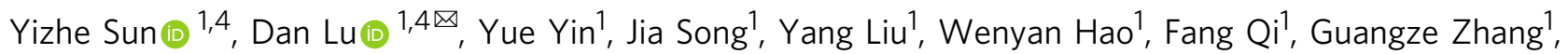
Xin Zhang ${ }^{1}$, Liang Liu', Zhiqiang Lin', Hui Liang ${ }^{1}$, Xuyang Zhao ${ }^{1}$, Yan $\operatorname{Jin}^{1} \&$ Yuxin Yin (1) 1,2,3凶

PTEN is frequently mutated in human cancers and PTEN mutants promote tumor progression and metastasis. PTEN mutations have been implicated in immune regulation, however, the underlying mechanism is largely unknown. Here, we report that PTEN $\alpha$, the isoform of PTEN, remains active in cancer bearing stop-gained PTEN mutations. Through counteraction of $\mathrm{CD}^{+} \mathrm{T}$ cell-mediated cytotoxicity, PTEN $\alpha$ leads to $\mathrm{T}$ cell dysfunction and accelerates immune-resistant cancer progression. Clinical analysis further uncovers that PTEN $\alpha$-active mutations suppress host immune responses and result in poor prognosis in cancer as relative to PTEN $\alpha$-inactive mutations. Furthermore, germline deletion of Pten $\alpha$ in mice increases cell susceptibility to immune attack through augmenting stress granule formation and limiting synthesis of peroxidases, leading to massive oxidative cell death and severe inflammatory damage. We propose that PTEN $\alpha$ protects tumor from T cell killing and thus PTEN $\alpha$ is a potential target in antitumor immunotherapy.

\footnotetext{
${ }^{1}$ Institute of Systems Biomedicine, Department of Pathology, School of Basic Medical Sciences, Beijing Key Laboratory of Tumor Systems Biology, Peking University Health Science Center, Beijing, China. ${ }^{2}$ Peking-Tsinghua Center for Life Sciences, Peking University Health Science Center, Beijing, China.

${ }^{3}$ Institute of Precision Medicine, Peking University Shenzhen Hospital, Shenzhen, China. ${ }^{4}$ These authors contributed equally: Yizhe Sun, Dan Lu.

凶email: taotao@bjmu.edu.cn; yinyuxin@hsc.pku.edu.cn
} 
T he importance of phosphatase and tensin homolog deleted on chromosome ten (PTEN) in tumorigenesis is underscored by its frequent mutations, including missense, nonsense, frameshift, and large deletions $s^{1,2}$. The tumorsuppressive activity of PTEN largely depends on its lipid phosphatase activity, which antagonizes PI3K/AKT activation ${ }^{1}$. Accordingly, a significant fraction of PTEN missense mutations is identified in human cancers, which selectively abolish its phosphatase activity ${ }^{3}$. Additionally, more than half of PTEN mutations result in protein truncation and instability, which are functionally comparable to PTEN loss ${ }^{1,4}$. In spite of the dysregulation of PI3K-AKT signaling in cancers with PTEN truncated or missense mutations, the outcomes of tumors with different types of PTEN mutations are distinct ${ }^{5,6}$. Therefore, an in-depth understanding of the biological functions of various PTEN mutations in tumorigenesis is still in need, and crucial for cancer treatment.

Through restoring or enhancing the effector function of $\mathrm{CD}^{+}$ tumor-infiltrating $\mathrm{T}$ cells, immunotherapy has revolutionized the treatment of patients with advanced-stage cancers 7 . However, this therapy is limited to a minority of patients with cancer, and the clinical efficacy is often compromised along with tumor development ${ }^{7,8}$. Previous studies have revealed that tumor cells can hijack strategies developed to limit immune responses, thereby affecting priming, activation, and recruitment of T cells, causing tumor immune escape ${ }^{9}$. Apart from these processes of $\mathrm{T}$ cells, effective anti-tumor immunity also requires cytotoxic $\mathrm{T}$ lymphocytes (CTLs)-mediated cell killing ${ }^{10}$. Researches have shown that the function of CTLs depends on the ROS production in the target cells, and recent studies further uncover that CTLs cause different types of cell death depending on the context of the target cells ${ }^{11-13}$. These phenomena lead to the hypothesis that, instead of affecting $\mathrm{T}$ cell function, tumor-intrinsic factors may directly affect the susceptibility of tumor cells to CTLs. To date, researches on tumor immune escape still mainly focus on the effects of tumor cells on $\mathrm{T}$ cells, while little is known about whether tumor cells can escape from CTLs-mediated killing through modulating their own metabolic state.

PTENa (also known as PTEN-L) is the first identified isoform of PTEN, which contains an additional disordered 173-amino acid $\mathrm{N}$ terminus ${ }^{14,15}$. This region confers functions of PTENa that are distinct from those of PTEN ${ }^{14,15}$. The role of PTENa in tumors is complicated, which limits tumor growth in glioblastoma cells, while promotes carcinogenesis in liver cancer ${ }^{4,16}$. Up to now, the effects of PTENa in anti-tumor immunity remain elusive. In this study, we find that PTENa acts as an immune suppressor that restricts $\mathrm{CD}^{+} \mathrm{T}$ cell response against PTEN mutant cancers, consequently resulting in poor outcome of the disease. Rather than directly affecting $\mathrm{T}$ cell priming or differentiation, PTENa promotes the cell resistance to T cell-mediated cytotoxicity and results in tumor immune escape. Reciprocally, loss of PTENa down-regulates translation of proteins associated with the oxidation-reduction process, and potentiates ferroptosis upon incubation with cytotoxic $\mathrm{T}$ cells, exacerbating inflammatory responses and promoting immune clearance. Our data thus demonstrate that PTENa acts as a suppressor of $\mathrm{T}$ cell cytotoxicity-induced oxidative cell death, and highlight the importance of targeting PTENa in antitumor immune therapy.

\section{Results}

PTENa remains active in PTEN-mutant cancer. Loss-offunction mutations in the PTEN gene are frequent in human cancer $^{3}$. In addition to phosphatase-inactive mutations, abundant stop-gained mutations were detected in exons encoding the C-terminal domain of PTEN (Fig. 1a). Through induction of
PTEN instability, stop-gained mutations restrict the function of phosphatase PTEN, whose effects are largely identical to those of phosphatase-inactive mutations ${ }^{1}$. However, through analyzing the survival of patients with tumor-bearing PTEN mutations, we found that the outcome of patients with stop-gained mutations was poorer than those with phosphatase-inactive mutations (Fig. 1b and supplementary Fig. 1a). We next analyzed differentially expressed genes in cancers with these two-type mutations based on the matched diagnosis age (40-60 years), aneuploidy score $(<3)$, and neoplasm histologic grade (G3). Utilizing gene set enrichment analysis (GSEA), we found that genes related to adaptive immune response were selectively enriched in cancers with phosphatase-inactive mutations as compared with those with stop-gained mutations (Fig. 1c). Similar results were also detected between the two-type mutations in a broader context (Fig. 1d). These results lead to the hypothesis that, aside from tumor-promoting effects, stop-gained mutations of PTEN restrict host immune response against cancer.

As an isoform of PTEN, PTENa is initially translated from a CUG codon upstream of and in-frame with the coding region of canonical PTEN ${ }^{15}$. As shown in Supplementary Fig. $1 \mathrm{~b}$, phosphatase-inactive mutations impaired the phosphatase activity of PTEN and its isoform PTENa. To assess the effects of stopgained mutations on PTEN isoforms, we analyzed the protein stability of the PTEN family with or without stop-gained mutations. As shown in Fig. 1e, f and Supplementary Fig. 1c, in contrast to the identical half-life between wild-type PTEN and PTENa, stop-gained mutations accelerated the degradation of PTEN rather than PTENa. To further confirm this result, we utilized the Jurkat and MOLT4 cells, which have been reported to carry stop-gained PTEN mutations ${ }^{17}$. In accordance with the bioinformatics data, we found that MOLT4 cells contain PTENK267Rfs*9 $^{*}$ mutation and Jurkat cells contain PTEN ${ }^{\text {R234Afs* }}$ and PTEN ${ }^{\mathrm{L} 247^{*}}$ mutations by DNA-sequencing analysis (Supplementary Fig. 1d). We then employed an anti-PTEN antibody to assess the status of endogenous PTEN and PTENa in Jurkat and MOLT4 cells. As shown in Supplementary Fig. 1d, compared with wild-type PTEN and PTENa in HEK-293T, truncated PTENa rather than PTEN can be detected in both Jurkat and MOLT4 cells. Our data thus indicate that PTENa remains active in cancer with stop-gained PTEN mutations (Supplementary Fig. 1b).

PTENa promotes tumor immune escape. To investigate the role of PTENa in tumorigenesis, we used CRISPR/Cas9 technology to knock out the endogenous PTEN in the B16-F10 cell line, which also abolished the expression of PTENa, and subsequently reenforced the expression of PTENa in Pten ${ }^{-/-}$cell line. As shown in Supplementary Fig. 2a-c, ectopic expression of PTENa elicited little effects on cell proliferation in vitro. In light of the impaired immune response in cancer with stop-gained mutations, we thus employed irradiated B16-F10 cell line as tumor vaccine to assess the role of PTENa in modulation of host immune response against cancer (Fig. 2a). As shown in Fig. 2b, in contrast to the identical volume of tumor with or without PTENa in unimmunized C57BL/6 mice, overexpression of PTENa weakened the effectiveness of cancer vaccination. Through analysis of tumorinfiltrating lymphocytes (TIL) by flow cytometry, less amounts of $\mathrm{CD} 45^{+}$immune cells were detected in PTENa-expressing tumors as relative to control tumors (Fig. 2c).

To study the status of immune cells in PTENa-expressing tumors, we applied scRNA-seq methods to investigate CD45 ${ }^{+}$ immune cells isolated from PTENa-expressing or control tumors with two replicates. To remove batch-effect, data integration and batch-effect correction were applied to the data. Utilizing graphbased clustering to analyze the tumor-infiltrating immune cells, 


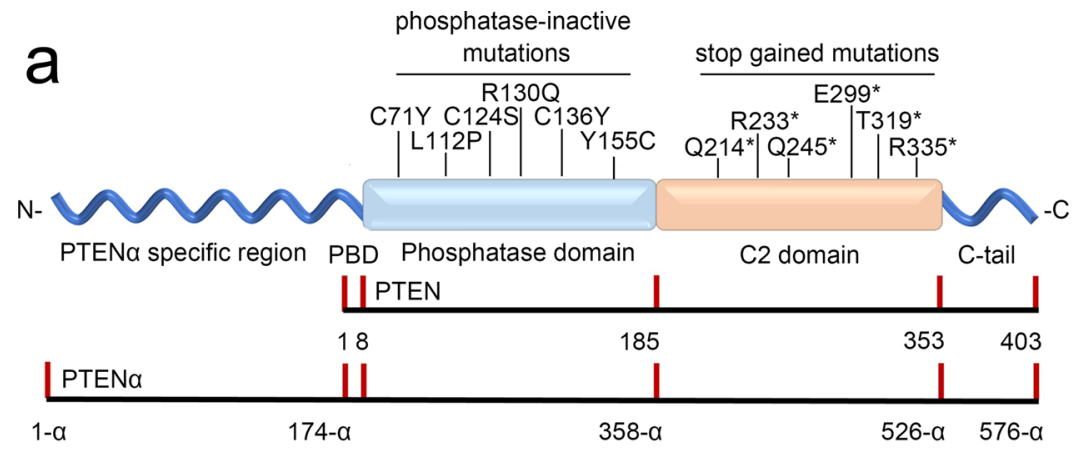

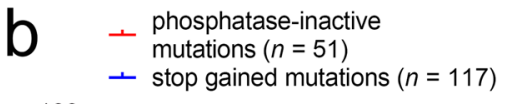

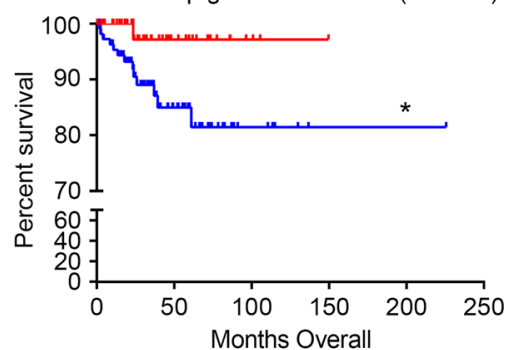

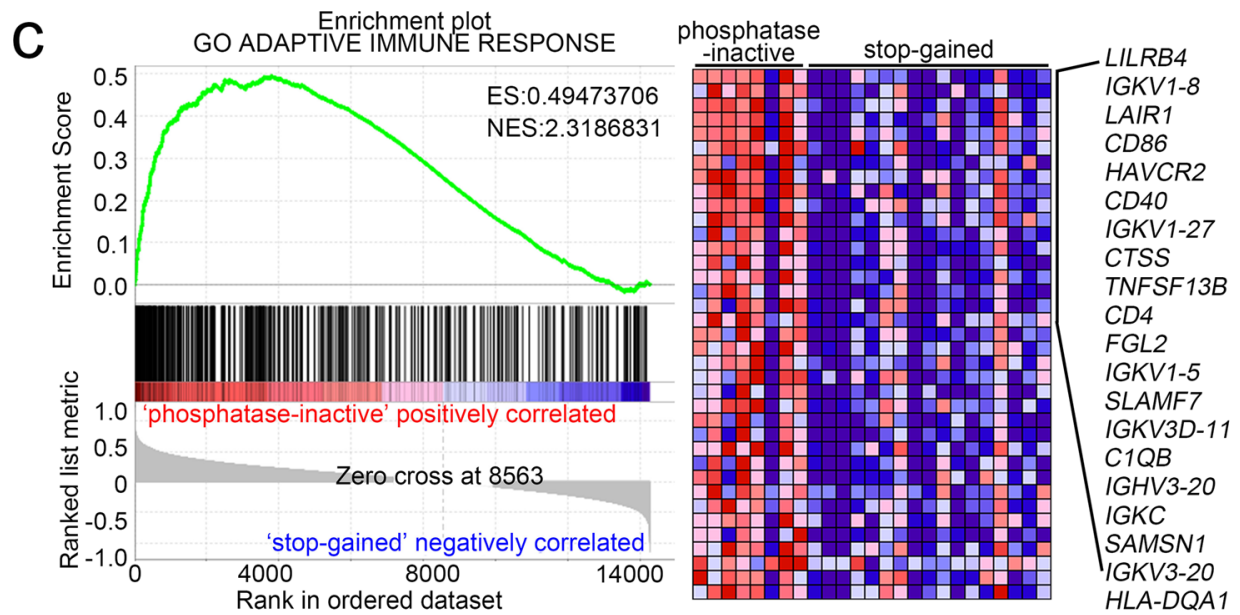
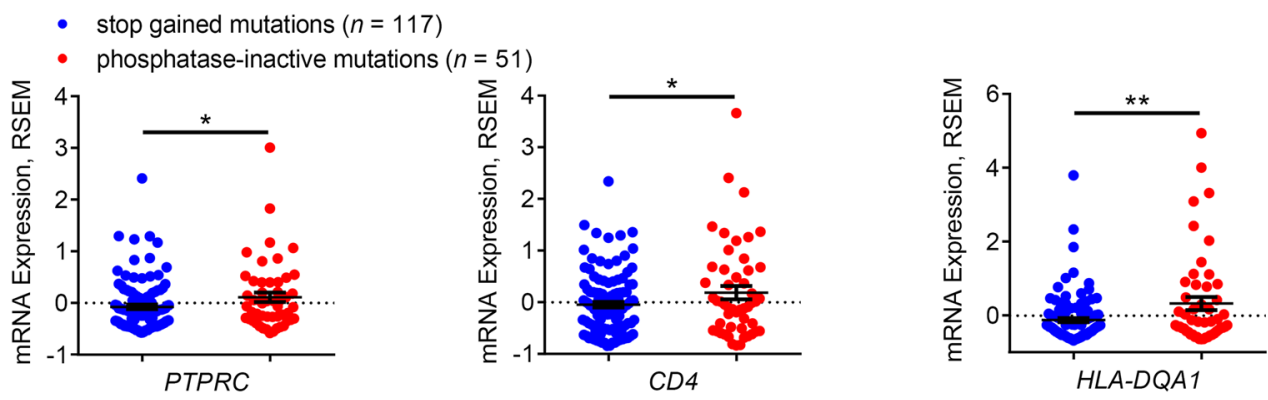

e

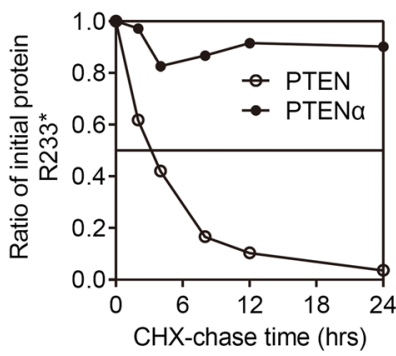

$f$

$\mathrm{CHX}(100 \mu \mathrm{g}) \quad 0 \quad$ h $4 \mathrm{~h}$ 8h $12 \mathrm{~h} 24 \mathrm{~h}(\mathrm{kDa})$
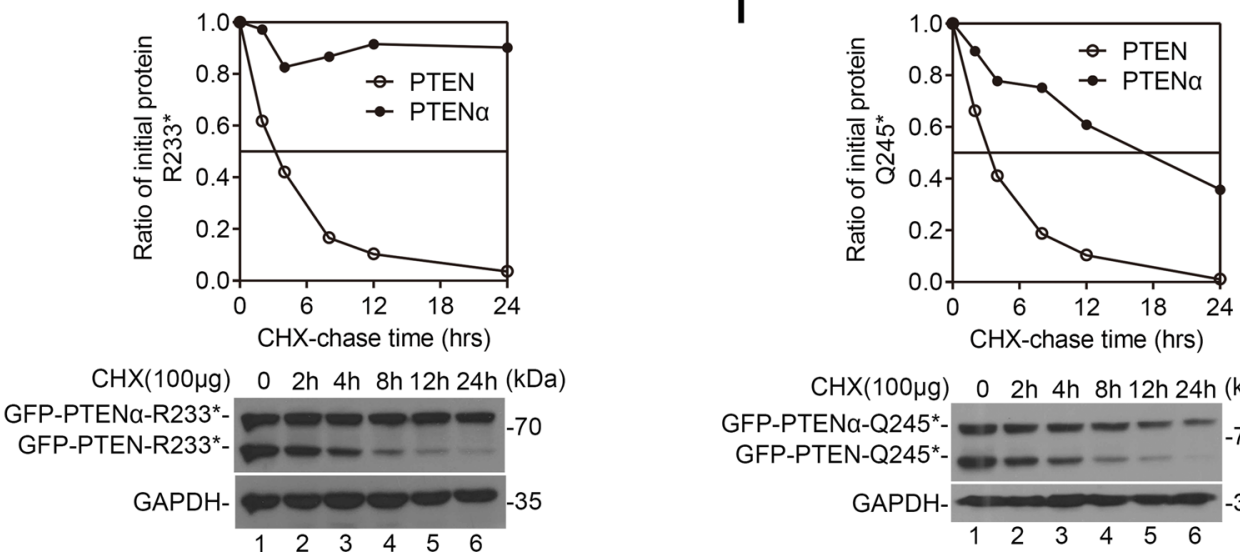

$\mathrm{CHX}(100 \mu \mathrm{g}) \quad 0 \quad 2 \mathrm{~h} 4 \mathrm{~h} 8 \mathrm{~h} 12 \mathrm{~h} 24 \mathrm{~h}(\mathrm{kDa})$

GFP-PTENa-Q245*- $=--70$ GFP-PTEN-Q245*-

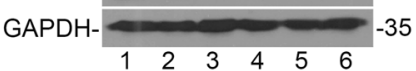

we identified 12 clusters of immune cells for $10 \times$ data. We then defined the clusters based on the exclusive expression of canonical marker genes. As shown in Supplementary Fig. 2d, e, and Supplementary Table 1, major immune cell types in cancers including $\mathrm{CD} 4^{+} \mathrm{T}, \mathrm{CD} 8^{+} \mathrm{T}$, Treg, $\mathrm{NK}$, monocyte, macrophages, neutrophil, B, plasma B, plasmacytoid dendritic cells ( $\mathrm{pDCs}$ ), and conventional DCs (cDCs). Compared with more than $80 \%$ of lymphoid cells in the control tumor, the percentage of $\mathrm{T}$ 
Fig. 1 PTEN $\alpha$ remains active in PTEN-mutant cancers. a Cartoons of structures of PTEN and PTEN $\alpha$. Domains that are contained by PTEN and PTEN $\alpha$ are shown in the simplified drawing, respectively. Stop gained mutations include all the nonsense or frameshift mutations terminated after $\mathrm{H}^{185}$ (last amino acid of phosphatase tensin-type domain of PTEN). Phosphatase-inactive mutations are from the UniProt database. Some of the two kinds of mutations are indicated in the graph. $\mathbf{b}$ Survival curve of uterine corpus endometrial carcinoma (UCEC) patients with PTEN mutations (Stop gained mutations, $n=117$; Phosphatase-inactive mutations, $\left.n=51,{ }^{\star} P=0.0377\right)$. Clinical data were acquired from the TCGA database. c GSEA of RNA-seq data of UCEC patients with matched diagnosis age (40-60 years), aneuploidy score $(<3)$, and neoplasm histologic grade (G3). The data were acquired from the TCGA database. ES is an abbreviation of Enrichment Score, and NES represents Normalized Enrichment Score. $\mathbf{d}$ Expression levels of immune surveillance-related genes in uterine corpus endometrial carcinoma (UCEC) patients with indicated PTEN mutations (Stop gained mutations, $n=117$; Phosphatase-inactive mutations, $n=51$, mean \pm s.e.m., $\left.{ }^{\star} P(P T P R C)=0.0400,{ }^{\star} P(C D 4)=0.0498,{ }^{\star \star} P=0.0022\right)$. e and $\mathbf{f}$ Immunoblot analysis of expression of GFP-tagged PTEN and PTEN $\alpha$ carrying indicated mutations in HEK293T cells treated with $100 \mu \mathrm{g} / \mathrm{ml} \mathrm{CHX}$ for indicated hours. An anti-GFP antibody was used. Gray values of PTEN and PTEN $\alpha$ relative to GAPDH were determined, using for the line chart. Statistical significance was assessed by Log-rank (Mantel-Cox) test (b) or two-tailed unpaired Student's $t$ test without multiple comparisons test (d). Data are representative of two (e and $\mathbf{f}$ ) independent experiments with similar results. Source data are provided as a Source Data file. See also Supplementary Fig. 1

lymphocytes and B lymphocytes were significantly reduced in PTENa-expressing tumors (Supplementary Fig. 2f). Considering the importance of $\mathrm{CD}^{+} \mathrm{T}$ cells in host antitumor immunity, we performed unsupervised clustering and identified four clusters for $\mathrm{CD}^{+} \mathrm{T}$ cells, each with its unique signature genes (Fig. $2 \mathrm{~d}$ and Supplementary Fig. 3a). In contrast to the other three clusters, the fourth cluster of CD8 ${ }^{+} \mathrm{T}$ cells, CD8T-Ctla4, expressed high levels of exhaustion markers Clta4, $P d c d 1$, and Lag3, thus representing exhausted CD8 ${ }^{+} \mathrm{T}$ cells (Fig. 2e). As expected, the percentage of C4_CD8T-Ctla4 was remarkably increased in PTENa-expressing tumors as compared with control tumors (Fig. 2f, g). Consistent with scRNA-seq results, subsequent flow cytometry revealed that overexpression of PTENa limited the amounts of infiltrated lymphocytes, and enhanced PD-1 and TIM-3 expression on $\mathrm{CD}^{+}$tumor-infiltrating $\mathrm{T}$ cells (supplementary Fig. $3 \mathrm{~b}-\mathrm{f}$ ). Furthermore, we also measured the production of IFN $\gamma$ in the tumor-infiltrating $\mathrm{CD}^{+} \mathrm{T}$ cells in the treatment of PMA/ ionomycin. As shown in Supplementary Fig. $3 \mathrm{~g}$, the production of IFN $\gamma$ was weakened in $\mathrm{CD}^{+} \mathrm{T}$ cells from PTENa-expressing tumors relative to those in control tumors.

To further confirm the immune regulatory role of PTENa in cancer, we performed the adoptive T-cell transfer assay. Mock or PTENa-expressing B16-Pten-1- cells were infected with LCMV$\mathrm{Cl13}$, and subcutaneously injected into nude mice. 10 days later, the activated $\mathrm{CD} 8+\mathrm{T}$ cells were harvested from spleen in mice infected with LCMV-Cl13 on day-7 post infection, and subsequently transferred into the tumor-bearing nude mice. Similar procedures were repeated on 14-day and 18-day in B16 tumorbearing mice (Fig. 2h). As shown in Fig. 2i, viral infection hardly influenced tumor growth of Mock or PTENa-expressing B16 cells in nude mice, and ectopic expression of PTENa elicited no effects on cell proliferation, which is consistent to the data in C57BL/6 mice (Fig. 2i). Moreover, the transferred $\mathrm{CD}^{+} \mathrm{T}$ cells exhibited significantly antitumor effects on Mock rather than PTENaexpressing B16 cells in nude mice (Fig. 2i). Notably, through analysis of $\mathrm{CD}^{+} \mathrm{T}$ cells infiltrating in spleen or tumors, we found that the amount of adoptive transferred $\mathrm{CD} 8^{+} \mathrm{T}$ cells were identical between the nude mice with PTENa-expressing B16 cells and those with Mock B16 cells (Supplementary Fig. 3h). Taken together, our data demonstrate that PTENa blocks T cellmediated cancer eradication and promotes cancer immune escape.

Furthermore, we also investigated the effects of PTENa bearing stop-gained mutations in cancer immune escape. Analog to the immunosuppressive effects of wild-type PTENa, overexpression of PTENaCTG-R233* also reduced the effectiveness of tumor vaccine and facilitated tumor growth (Supplementary Fig. 3i). To ascertain whether the immunosuppressive role of PTENa is dependent on its secretion, we also employed the vector of PTENaCTG- $\triangle 6 \mathrm{~A}$ that has been reported to abolish secretion of
PTENa ${ }^{14}$. Utilizing the cancer vaccine model, we found that overexpression of PTENaCTG- $\Delta 6 \mathrm{~A}$ also promoted cancer immune escape, which is identical to the effects of PTENaCTG-R233*, indicating that the secretion characteristics of PTENa hardly affects its immunoregulatory function (Supplementary Fig. 3i). Considering that PTENa mutations also exhibit clinical relevance in colon cancers, we employed CT26 cells (murine colorectal cancer cell line) to assess the role of PTENa in tumor vaccination model. We first used shRNA to knock down the endogenous PTEN expression in CT26 cells, and subsequently stably transfected with the vector encoding Mock or PTENa (Supplementary Fig. 3j). In accordance with the data in B16 cells, ectopic expression of PTENa elicited immunosuppressive effects and promoted tumor development in immunized mice (supplementary Fig. 3k). Together, our data thus indicate that the presence of PTENa in cancers subverts host immune attack.

PTENa promotes $\mathrm{T}$ cell exhaustion in a $\mathrm{T}$ cell-extrinsic manner. Since the presence of PTENa in cancers drives $\mathrm{CD}^{+} \mathrm{T}$ cell dysfunction, we employed Pten $\alpha^{-/-}$mice to further study the role of PTENa in the modulation of T cell fate. Although PTENa was expressed in both lymphoid and non-lymphoid organs (Supplementary Fig. 4a), Pten $\alpha^{-/-}$mice developed normally, and no spontaneous tumor was detected within 2-year old. In order to determine whether loss of PTENa affects the proliferation, differentiation, and survival of $\mathrm{T}$ cells, we firstly employed a CFSEstaining assay to measure the $\mathrm{T}$ cell expansion during activation. As shown in Supplementary Fig. 4b, c, deletion of PTENa elicited little effects on $\mathrm{T}$ cell expansion during $\mathrm{T}$ cell priming. Besides, identical ratio of iTreg cell $\left(\mathrm{CD} 4^{+} \mathrm{CD} 25^{+} \mathrm{Foxp}^{+}\right)$, Th1 $\left(\mathrm{CD} 4^{+} \mathrm{IFN} \gamma^{+}\right)$, Th17 $\left(\mathrm{CD}^{+}{ }^{\mathrm{IL}}-17 \mathrm{~A}^{+}\right)$and $\operatorname{Tr} 1\left(\mathrm{CD} 4^{+} \mathrm{IL}-10^{+}\right)$cells were induced under various $\mathrm{T}$-cell polarizing conditions between wild-type and Pten $\alpha^{-/-}$mice (Supplementary Fig. 4d). Finally, we used Annexin $\mathrm{V} / 7-\mathrm{AAD}$ staining to assess cell death during $\mathrm{T}$ cell activation. As shown in Supplementary Fig. 4e, no significant difference was detected between wild-type and Pten $\alpha^{-1-} \mathrm{T}$ cells.

We next utilized lymphocytic choriomeningitis virus clone 13 (LCMV-Cl13) to induce T cell exhaustion. As shown in Fig. 3a, b, high viral loads were found in the lungs in wild-type mice, whereas Pten $\alpha^{-1-}$ mice showed a much-reduced viral burden on day 30 post infection. Compared with wild-type mice, the frequency of virus-specific CD8 ${ }^{+} \mathrm{T}$ cells $\left(\mathrm{GP}_{33-41}{ }^{+}\right)$was increased in the lungs from Pten $\alpha^{-/-}$mice (Fig. $3 \mathrm{c}$ and Supplementary Fig. 5a). Consistent with published studies ${ }^{18}$, we observed that high levels of inhibitory receptors including PD-1, TIM-3, and LAG-3 were expressed on the surface of virus-specific wild-type $\mathrm{CD}^{+}{ }^{+} \mathrm{T}$ cells rather than Pten $\alpha^{-/-} \mathrm{CD} 8^{+} \mathrm{T}$ cells during chronic infection (Fig. 3d). Moreover, we stimulated CD8 ${ }^{+} \mathrm{GP}_{33-41}{ }^{+} \mathrm{T}$ cells with virus-specific peptides to induce the production of effector cytokines in wild-type and Pten $\alpha^{-/-} \mathrm{T}$ cells. As shown in 

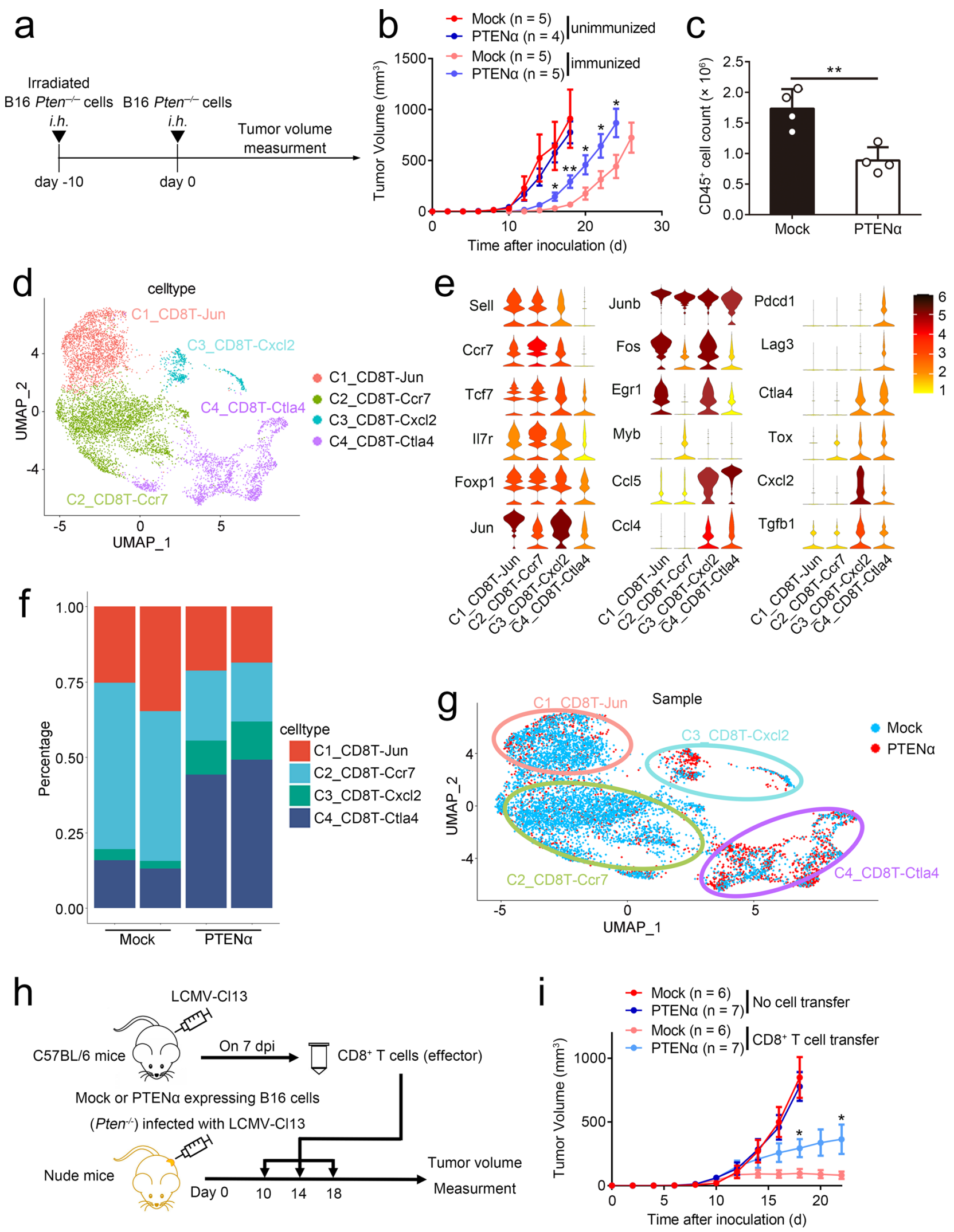

Supplementary Fig. 5b, Pten $\alpha^{-/-} \mathrm{T}$ cells produced greater amounts of effector cytokines than wild-type $\mathrm{T}$ cells upon exposure to viral peptide. Similar results were also observed in virus-specific $\mathrm{T}$ cells from spleens (Supplementary Fig. $5 \mathrm{c}-\mathrm{e}$ ). These data thus indicate that deletion of PTENa blocks $\mathrm{T}$ cell dysfunction.
We next sought to determine whether $\mathrm{T}$ cell polarization is dependent on T cell-intrinsic PTENa. To this end, we used a bone marrow (BM) transplantation model in which Pten $\alpha^{-/-}$or wildtype mice were lethally irradiated and transplanted with wild-type or Pten $\alpha^{-/}$BM and subsequently infected with LCMV-Cl13. As shown in Fig. 3e, on day 30 post-infection, the numbers of virus- 
Fig. 2 PTEN $\alpha$ promotes tumor immune escape. a-g Cancer vaccination model of Mock or PTEN $\alpha$ transfected Pten ${ }^{-/-}$B16 cells. a Schematic diagram of the cancer vaccine model. $\mathbf{b}$ Tumor volumes of $\mathrm{C} 57 \mathrm{BL} / 6$ mice subjected to the cancer vaccination model were monitored overtime. The unimmunized group refers to mice without irradiated B16 cells immunization. The statistical significances between the immunized groups were indicated (PTEN $\alpha$ unimmunized, $n=4$ mice; other groups, $n=5$ mice, mean \pm s.e.m., ${ }^{\star} P(\mathrm{~d} 16)=0.0254,{ }^{\star} P(\mathrm{~d} 20)=0.0339,{ }^{\star} P(\mathrm{~d} 22)=0.0487,{ }^{\star} P(\mathrm{~d} 24)=0.0450$, ${ }^{\star \star} P=0.0077$ ). c The tumor-infiltrating lymphocytes (TILs) were isolated when the tumor volumes of the Mock-immunized group reach $200 \mathrm{~mm}^{3}$, and the cells were subjected to flow cytometry analysis. The cell counts of CD $45^{+}$cells were used for statistical analysis $\left(n=4\right.$ mice, mean \pm SD, $\left.{ }^{\star \star} P=0.0044\right)$. Gating strategies were shown in Supplementary Fig. 3b. d-g TILs were isolated when the tumor volumes of Mock-immunized group reach $200 \mathrm{~mm}^{3}$, and CD45 ${ }^{+}$cells were sorted, using for 10× scRNA-seq with two replicates. 25,098 cells were identified as 12 clusters utilizing graph-based clustering. d Cd8T cells were further analyzed and identified as 4 clusters utilizing graph-based clustering. e Violin plot of differentiated genes in the 4 clusters of Cd8T cells. The color of each violin is the average expression value of a given gene. $\mathbf{f}$ and $\mathbf{g}$ Umap and proportion of the 4 clusters of Cd8T cells were shown. $\mathbf{h}$ and $\mathbf{i}$ Adoptive T cell transfer model of Mock or PTEN $\alpha$ expressing B16-Pten ${ }^{-1-}$ cells. $\mathbf{h}$ An illustration of mice treatment. $\mathbf{i}$ Tumor volumes was monitored over time (Mock, $n=6$ mice; PTEN $\alpha, n=7$ mice, mean \pm s.e.m., $\left.{ }^{\star} P(\mathrm{~d} 18)=0.0360,{ }^{\star} P(\mathrm{~d} 22)=0.0481\right)$. Statistical significances between $\mathrm{CD} 8{ }^{+}$ T cells transferred groups were shown. Statistical significance was assessed by a two-tailed unpaired Student's $t$ test without multiple comparisons test $(\mathbf{b}, \mathbf{c}, \mathbf{i})$. Data are representative of two $(\mathbf{b}$ and $\mathbf{i})$ or three $(\mathbf{c})$ independent experiments with similar results. Source data are provided as a Source Data file. See also Supplementary Figs. 2 and 3.

specific T cells were identical between $P t e n \alpha^{-/-}$recipients of wildtype BM and those of Pten $\alpha^{-/-}$BM. Of note, compared with wildtype donor mice, greater amounts of virus-specific $\mathrm{T}$ cells were detected in the two types of Pten $\alpha^{-1-}$ recipient mice (Fig. 3e). Conversely, LCMV-Cl13 infection resulted in lower amounts of virus-specific $\mathrm{T}$ cells in wild-type recipients, no matter which kind of BM is adoptive transferred (Fig. 3f). Consistent results were also detected by assessment of inhibitory receptor expression and effector cytokine production (Supplementary Fig. 6a-d). Combined with the data that PTENa is also expressed in nonlymphoid organs (Supplementary Fig. 4a), our data thus demonstrate that $\mathrm{T}$ cell-extrinsic PTENa drives T cell exhaustion.

PTENa promotes cell resistance to $T$ cell cytotoxicity. To investigate the mechanism by which PTENa promotes $\mathrm{T}$ cell exhaustion, we analyze the epithelial-immune cell interaction. As shown in Fig. 4a, loss of PTENa triggered stronger inflammatory response in the early stage of higher titers of LCMV-Cl13 infection, causing massive congestion and edema in the lungs of Pten $\alpha^{-/-}$ mice upon viral infection. Moreover, greater amounts of death cells were detected in Pten $\alpha^{-/}$lungs assessed by TUNEL staining (Fig. 4b). Besides, we performed mass spectrometry analysis of tissue interstitial fluid (TIF) in the lungs during inflammation, and found that a series of nuclear proteins were released in extracellular matrix of lungs from Pten $\alpha^{-/}$mice at the early stage of viral infection, which acts as danger-associated molecular patterns (DAMPs) and activates immune response (Fig. 4c). Consistently, loss of PTENa increased the amounts of HSP70, HSP90, HMGB1, and eATP (extracellular ATP) in TIF in the lung during viral infection (Figs. 4d, e). These results thus indicate that deletion of PTENa promotes immunogenic cell death upon LCMV infection.

Since LCMV-Cl13 is a non-cytolytic virus that hardly induces cell death in vitro ${ }^{19}$, we hypothesized that $\mathrm{T}$ cell-mediated cytotoxicity mainly contributed to the severe cell death. To this end, we performed in vitro killing assay to investigate whether PTENa affects target cell responses to CTLs. We pulsed Mock or PTENa expressing B16-Pten ${ }^{-/}$cells with LCMV-GP $_{33-41}$ peptide and incubated with $\mathrm{CD}^{+}{ }^{+} \mathrm{T}$ cells isolated from spleens of C57BL/6 mice untreated or infected with LCMV-Cl13. Notably, incubation of $\mathrm{CD}^{+} \mathrm{T}$ cells from the infected mice induced significantly lower cell lysis rates in PTENa-expressing B16 cells relative to those control cells (Fig. 4f). Moreover, compared with PTENa-expressing cells, the $\mathrm{T}$ cell-mediated cytotoxicity of Mock B16-Pten ${ }^{-/}$- cells induced high level of ATP release and intracellular ROS accumulation, which indicated that loss of PTENa resulted in non-apoptotic cell death under immune attack (Fig. 4g, h). Similar results were also detected in Pten $\alpha^{+/+}$and Pten $\alpha^{-1-}$ MEFs (Supplementary Fig. 7a-c). Our data thus demonstrate that CTLs triggers immunogenic death of Pten $\alpha^{-/}$cells that exacerbates inflammatory damage upon exposure to pathogens. The existence of PTENa correspondingly limits antigen clearance, resulting in $\mathrm{T}$ cell exhaustion.

PTENa restricts oxidative cell death. Previous studies have revealed that ROS is essential for CTLs-mediated cytotoxicity ${ }^{11}$. In consideration of the higher ROS level in PTENa-deletion cells induced by CTLs, we treated Pten $\alpha^{+/+}$and Pten $\alpha^{-/-}$MEFs with hydrogen peroxide $\left(\mathrm{H}_{2} \mathrm{O}_{2}\right)$ to mimic CTLs stimulation and investigated the nature of cell death caused by PTENa deficiency. As shown in Supplementary Fig. 8a, b, PTENa deficiency markedly increased cell susceptibility to ROS-mediated cell death. Moreover, using a series of cell death inhibitors, we found that the ferroptosis inhibitor ferrostatin-1 (Fer-1) was the only one completely abolishing the cell death induced by $\mathrm{H}_{2} \mathrm{O}_{2}$ (Supplementary Fig. 8c). Consistent results were also observed in Mock or PTENa-expressing Pten ${ }^{-/}$B16 cells (Fig. 5a-c). Besides, treatment of iron chelator deferomamine (DFO) and $\alpha$-TOC (a most abundant form of vitamin E) significantly rescued Pten $\alpha^{-/-}$ MEFs from $\mathrm{H}_{2} \mathrm{O}_{2}$-induced cell death (Supplementary Fig. 8d, e). Reciprocally, supplementation of the ferroptosis activator erastin and RSL3 significantly promoted cell death compared with other cell death agonists did (Supplementary Fig. 8f, g). To further confirm that loss of PTENa selectively induces ferroptosis, we measured the status of GPX4, which is a key regulator of ferroptosis. As shown in Supplementary Fig. 8h, the protein levels of GPX4 were reduced in lungs from Pten $\alpha^{-1-}$ mice compared with wild-type mice upon exposure to the virus.

In order to ascertain that suppression of ferroptosis mainly contributes to the oncogenic function of PTENa, we used $a$-TOC which is a radical scavenger and lipid peroxidation inhibitor, to suppress ferroptosis commitment. Considering only a portion of a-TOC dosage can reach the tumor by oral administration and its water-insoluble characteristic, we exploited a molecular-matched strategy to prepare $\alpha$-TOC-loading TPGS nanoparticles (NP-VE) with extremely high drug loading levels (up to $10 \mathrm{mg} / \mathrm{ml})^{20}$, which can effectively limit ROS production (Supplementary Fig. $8 \mathrm{i}, \mathrm{j}$ ). In vitro assays showed that $\alpha$-TOC treatment weakened the difference of cell death between PTENa-expressing cells and controls cells exposure to cytotoxic T cells (Fig. 5d). Besides, we also used BODIPY-C11 dye to analyze the level of lipid ROS. Analog to the intracellular ROS level, the presence of PTENa limited the level of lipid ROS in B16 cells $\left(\right.$ Pten $\left.^{-/}\right)$under T cell attack (Fig. 5e). Moreover, treatment of NP-VE in the cancer vaccine model promoted the progression of Mock-expressing B16-Pten ${ }^{-1-}$ cells to a similar level of PTENa expressing B16Pten $^{-1-}$ cells (Fig. 5f). Flow cytometry analysis of the infiltrating 


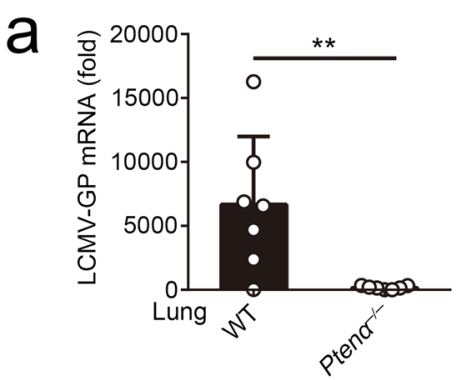

C
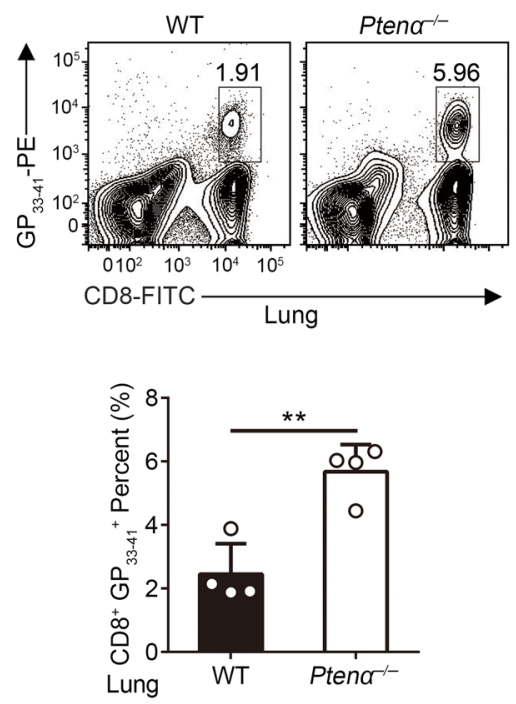

e

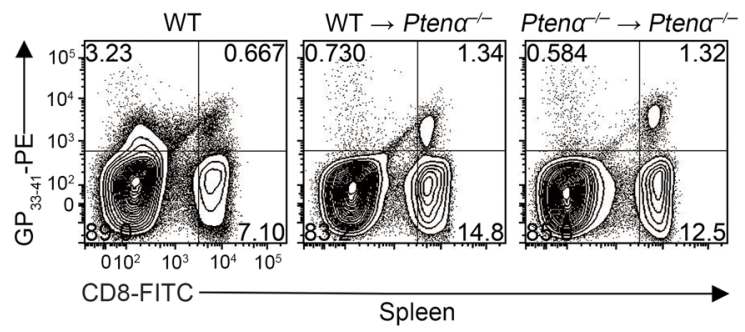

f

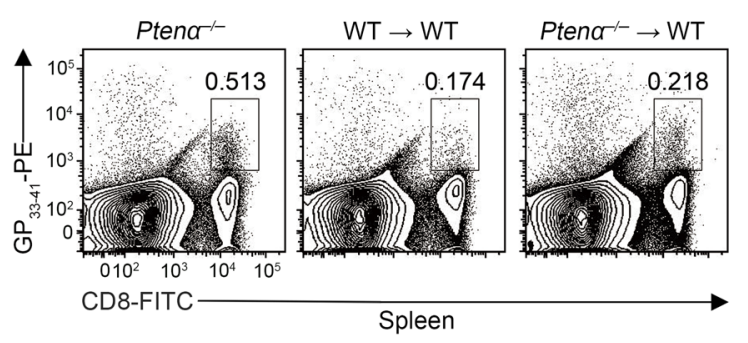

d
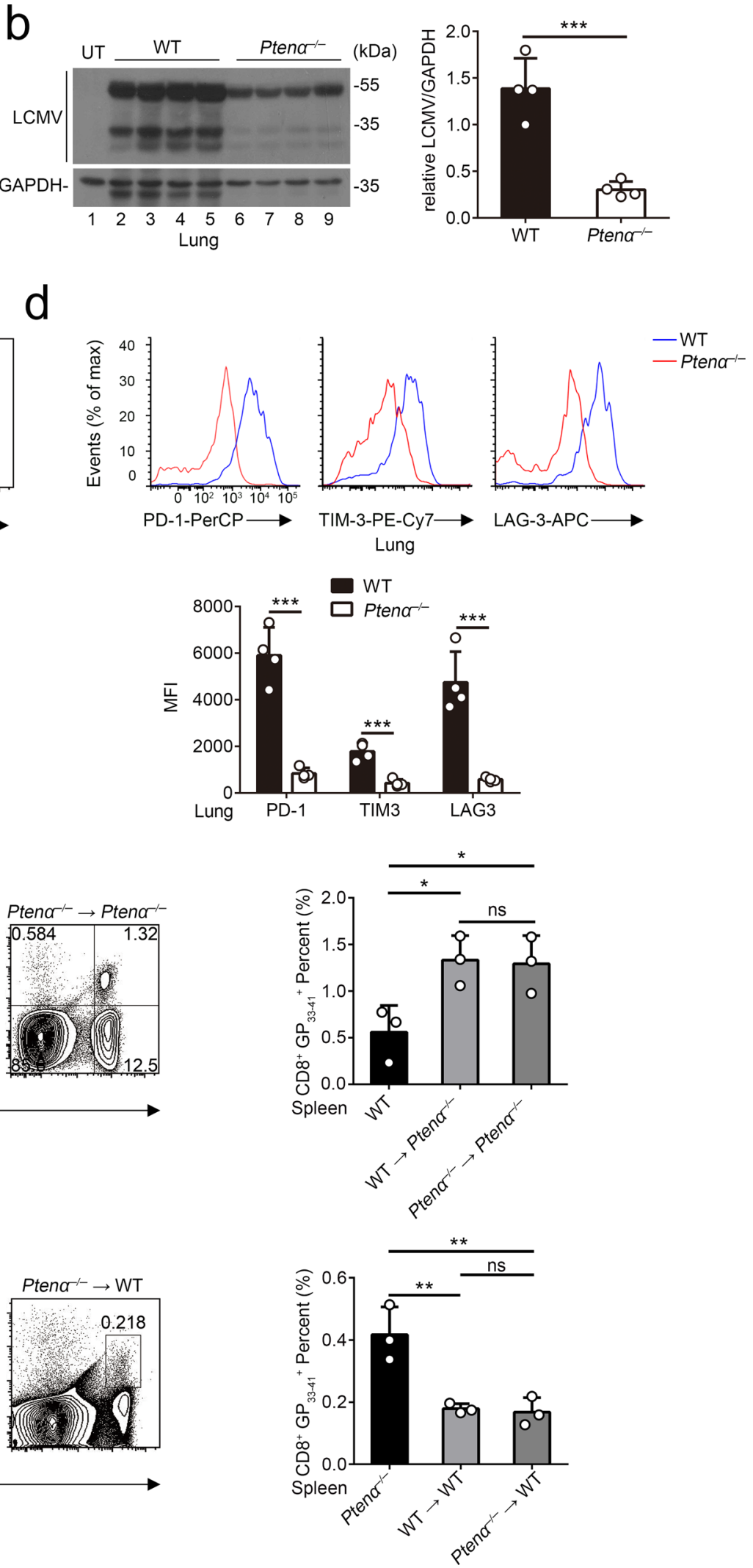

immune cells revealed that injection of NP-VE restricted the amount of $\mathrm{CD}_{4} 5^{+}$immune cells infiltrating in tumors as compared with untreated Mock tumors (Supplementary Fig. 9a). Besides, the presence of PTENa exhibited little effects on the recruitment of immune cells in tumors treated with NP-VE (Supplementary Fig. 9a), suggesting that blockade of oxidative cell death is essential for the immunosuppressive effects of PTENa. Further flow cytometry analysis demonstrated that NP-VE treatment or PTENa presence restricted both $\mathrm{T}$ and $\mathrm{B}$ cell recruitment in tumors (Supplementary Fig. 9b, c). In accordance with the suppressive effects of NP-VE treatment on $\mathrm{T}$ cell recruitment, increased expression of PD1 and reduced production 
Fig. 3 T cell-extrinsic PTENo promotes exhausted T cell formation. a-d Wild-type and Pten $\alpha^{-/-}$mice were i.v. infected with $5 \times 10^{5}$ PFU LCMV-CI13. On day 30 post-infection, the mice were sacrificed, and the lungs of the mice were harvested. $\mathbf{a}, \mathbf{b}$ The mRNA and protein levels of LCMV-GP in the lungs were analyzed with GRT-PCR (a) ( $n=7$ mice, mean \pm SD, $\left.{ }^{\star \star} P=0.0071\right)$ or immunoblot analysis (b) with anti-LCMV antibody, respectively. Gray values of LCMV proteins were determined, using for statistical analysis $(\mathbf{b})\left(n=4\right.$ mice, mean $\left.\pm S D,{ }^{\star \star \star} P=0.0007\right)$. UT untreatment. $\mathbf{c}$ Lymphocytes isolated from lungs of wildtype and Pten $\alpha^{-/-}$mice on day 30 post-infection were subjected to flow cytometry, and the frequencies of $\mathrm{CD}^{+} \mathrm{GP}_{33-41}{ }^{+}$cells were analyzed $(n=4$ mice, mean $\pm S D$, ${ }^{\star \star} P=0.0023$ ). Gating strategies were shown in Supplementary Fig. 5 a. $\mathbf{d}$ Flow cytometric analysis of the expression of PD-1, TIM-3 and LAG-3 on $\mathrm{CD}^{+}{ }^{-\mathrm{GP}_{33-41}+}$ cells from lungs of wild-type and Pten $\alpha^{-/-}$mice on day 30 post-infection $\left(n=4\right.$ mice, mean $\pm \mathrm{SD},{ }^{\star \star \star} P(\mathrm{PD}-1)=0.0002,{ }^{\star \star \star} P(\mathrm{TIM}-3)=0.0005$, ${ }^{\star \star \star} P($ LAG-3) $=0.0007)$. Gating strategies were shown in Supplementary Fig. $5 a$. e Bone marrow from wild-type and Pten $\alpha^{-/-}$mice were transplanted into Pten $\alpha^{-/-}$mice, respectively. 30 days after transplantation, mice were i.v. infected with $5 \times 10^{5}$ PFU LCMV-Cl13. On day 30 post-infection, spleens were harvested from the mice, and lymphocytes were isolated. Percentages of $\mathrm{CD} 8+\mathrm{GP}_{33-41}+$ cells were determined by flow cytometry analysis $(n=3$ mice, mean \pm SD, ns not significant, ${ }^{\star} P\left(\right.$ WT vs. WT $\rightarrow P$ ten $\left.\alpha^{-/-}\right)=0.0373,{ }^{\star} P\left(\right.$ WT vs. Pten $\alpha^{-/-} \rightarrow P$ ten $\left.\left.\alpha^{-/-}\right)=0.0456\right)$. Gating strategies were shown in Supplementary Fig. 5a. Unstained refers to samples that are unstained with PD-1, TIM-3, or LAG3. f Wild-type mice were adoptive transferred with BM from wild-type or Pten $\alpha^{-/-}$mice, and infected with $5 \times 10^{5}$ PFU LCMV-CI13 on day 30 post-transplantation. 30 days post-infection, splenocytes were harvested from the mice, and the percentages of $\mathrm{CD} 8^{+} \mathrm{GP}_{33-41}{ }^{+}$cells were determined by flow cytometry analysis $\left(n=3\right.$ mice, mean $\pm \mathrm{SD}, \mathrm{ns}$, not significant, ${ }^{\star \star} P\left(P t e n \alpha^{-/}-\mathrm{vs}\right.$. $\mathrm{WT} \rightarrow \mathrm{WT})=0.0061{ }^{\star \star}{ }^{\star} P\left(P \operatorname{ten} \alpha^{-/-}\right.$vs. Pten $\left.\left.\alpha^{-/-} \rightarrow \mathrm{WT}\right)=0.0049\right)$. Gating strategies were shown in Supplementary Fig. 5a. Statistical significance was assessed by two-tailed unpaired Student's $t$ test (a-d) or one-way ANOVA followed by Tukey's multiple comparisons test (e and $\mathbf{f}$ ). Data are representative of two (a-f) independent experiments. Source data are provided as a Source Data file. See also Supplementary Figs. 4-6.

of IFN $\gamma$ were also detected in $\mathrm{CD}^{+} \mathrm{T}$ cells isolated from mock tumors treated with NP-VE (Supplementary Fig. 9d, e). Taken together, our data demonstrate that PTENa increases cell resistance to CTLs-induced immunogenic cell death and consequently leads to cancer immune escape.

PTENa blocks stress granules formation during oxidative stress. To investigate the molecular mechanism by which PTENa modulates cell response to immune attack, we performed pulldown assays with FLAG-tagged proteins coupled with mass spectrometry under the condition of $\mathrm{H}_{2} \mathrm{O}_{2}$ treatment. Interestingly, we noticed that majority of PTENa-associated proteins were involved in stress granules assembly (G3BP1, G3BP2, etc.) and formation (eIF2 $\alpha$, PCBP1, PCBP2, ZC3HAV1, etc.) (Fig. 6a). Subsequent co-immunoprecipitation and immunofluorescence assays further confirmed the association of PTENa with stress granules components (Fig. 6b, c). To determine whether PTEN also participates in this process, we co-transfected PTEN or PTEN $\alpha$ with eIF2 $\alpha$ in the presence or absence of $\mathrm{H}_{2} \mathrm{O}_{2}$ treatment. Compared with the stimulatory effect of $\mathrm{H}_{2} \mathrm{O}_{2}$ treatment on the association of PTENa with eIF2a (Fig. 6d, lane 3-4 versus lane $1-2)$, PTEN did not interact with stress granules-associated proteins (Fig. 6d, lane 7-8 versus lane 5-6 and Fig. 6e, lane 3 versus lane 1 and 2 ).

To determine whether PTENa influences stress granules formation, we transfected mock or PTENa into Pten ${ }^{-1-}$ B16-cells followed by stimulating with $\mathrm{H}_{2} \mathrm{O}_{2}$, which is also a known inducer of stress granules. In spite of extensive co-localization of PTENa with intrinsic G3BP1 detected by immunofluorescence assay, overexpression of PTENa significantly inhibited $\mathrm{H}_{2} \mathrm{O}_{2}$-induced cytoplasmic foci formation compared with cells transfected with empty vectors (Fig. 6f, g). Together, these results indicate that PTENa selectively interacts with stress granules-associated proteins and blocks stress granules formation under stress conditions.

PTENa maintains protein synthesis of peroxidases. The phosphorylation of eIF $2 \alpha$ is crucial for stress granules formation ${ }^{21}$. Given that PTENa possesses the canonical phosphatase activity and interacts with eIF $2 \alpha$, we hypothesized that PTENa modulates eIF2 $\alpha$ phosphorylation under multiple kinds of stress conditions. As anticipated, stimulated by $\mathrm{H}_{2} \mathrm{O}_{2}$, overexpression of PTENa in Pten $^{-/-}$B16 cells inhibited eIF2 $\alpha$ phosphorylation (Fig. 7a, lanes 4 and 6 versus lanes 3 and 5). Similar results were also observed in MEFs (Supplementary Fig. 10a, lanes 4 and 6 versus lanes 3 and 5).
Additionally, unlike PTENa, enforced expression of PTEN or the phosphatase-dead PTENa-C297S mutant did not significantly influence the eIF2 $\alpha$ phosphorylation status in Pten $^{-/}$MEFs upon $\mathrm{H}_{2} \mathrm{O}_{2}$ exposure (Supplementary Fig. 10a, lanes 4 and 6 versus lanes 3 and 5). Besides, we also performed in vitro phosphatase assay and found that PTENa directly dephosphorylates eIF2 $\alpha$ in vitro (Fig. 7b, lane 3 versus lane 2). Our data thus confirm that PTENa acts as a phosphatase on eIF $2 \alpha$.

Since eIF2 $\alpha$ phosphorylation restricts host translational machinery ${ }^{21}$, we incubated Pten $\alpha^{+/+}$and Pten $\alpha^{-/-}$MEFs in medium containing ${ }^{35} \mathrm{~S}$ isotope-labeled methionine $\left({ }^{35} \mathrm{~S}-\mathrm{Met}\right)$ and then measured ${ }^{35} \mathrm{~S}$-labeled protein synthesis. Relative to Pten $\alpha^{+/+}$MEFs, a substantial decrease in protein synthesis was observed in Pten $\alpha^{-/}$MEFs after exposure to $\mathrm{H}_{2} \mathrm{O}_{2}$ or TG (Fig. 7c). Consistent with these findings, puromycin incorporation (as a measure of protein synthesis) into nascent cells was also significantly decreased under stress conditions in Pten $\alpha^{-/-}$MEFs compared with Pten $\alpha^{+/+}$MEFs, as revealed by immunofluorescence and immunoblotting analysis (Supplementary Fig. 10b, lanes 4-6 versus lanes 1-3, and Supplementary Fig. 10c). Similar results were also detected in Mock or PTENa-expressing Pten ${ }^{-/-}$ B16 cells (Fig. 7d, e, lanes 4-6 versus lanes 1-3). Together, our data thus indicate that deletion of PTENa promotes eIF2 $\alpha$ mediated translation inhibition.

Stress granules are known to be related with selective translation $^{22-24}$. To determine which proteins are regulated by eIF2 $\alpha$ phosphorylation and consequent stress granules assembly in Pten $\alpha^{-/-}$MEFs, we performed a mass spectrometry analysis of Pten $\alpha^{+/+}$and Pten $\alpha^{-/-}$MEFs under oxidative stress. As shown in Fig. 7f and Supplementary Fig. 10d, loss of PTENa selectively affected the synthesis of proteins associated with translation and oxidation-reduction processes. Ensued immunoblotting analysis confirmed that protein level of GPX4 rather than its mRNA level was significantly decreased in PTENa-null cells under oxidative stress condition (Fig. 7g, lane 4 and lane 6 versus lane 3 and lane 5, Supplementary Fig. 10e, lane 4 and lane 6 versus lane 3 and lane 5, and Supplementary Fig. 10f). Accordingly, intracellular ROS levels in $\mathrm{H}_{2} \mathrm{O}_{2}$-treated PTENa-expressing B16 cells were diminished relative to control cells (Fig. 7h).

To further confirm the essential role of Gpx4 in PTENamediated tumor immune escape, we used shRNA to knock down the endogenous Gpx4 expression in Pten ${ }^{-/-}$B16 cells, which was subsequently stable transfected with PTENa. Employing the murine tumor vaccination model, loss of Gpx4 neutralized the immunosuppressive effects of PTENa and remarkably increased 
a

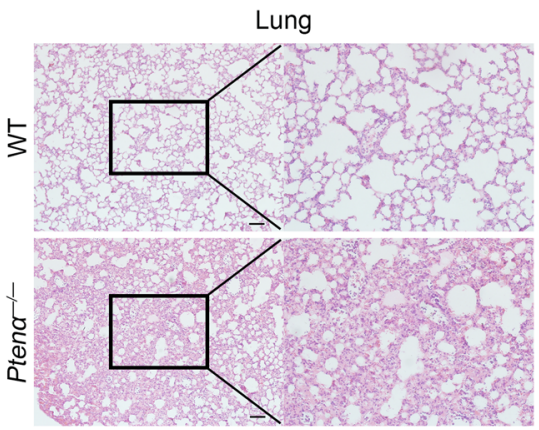

Enrichment plot:

GO Protein localization to nucleus

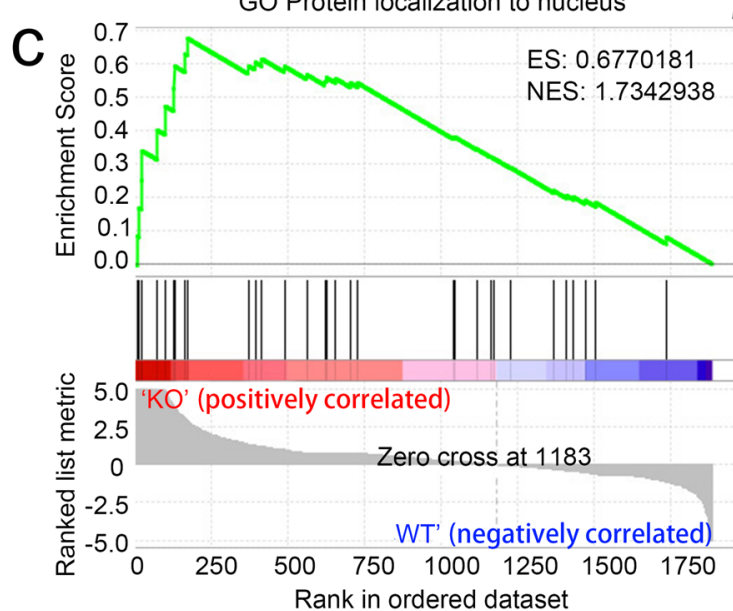

Ptena $^{\prime-}$ WT

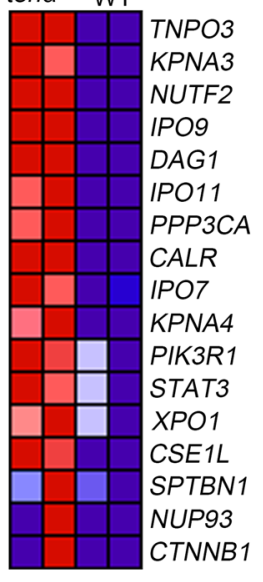

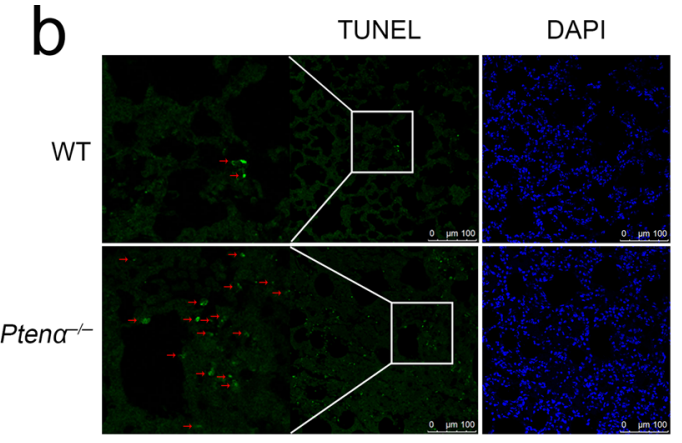

Lung
C Ratio: $\frac{\text { UT }}{1.01 .2} \frac{\text { WT }}{1.21 .0} \frac{\text { Ptend }^{\prime-}}{2.21 .7}$ (kDa)

Hsp90- - - - -100

Ratio: 1.01 .12 .11 .73 .02 .8

Hsp70- - - -70

Ratio: 1.01 .51 .41 .42 .42 .6

HMGB1- - - - - - -25

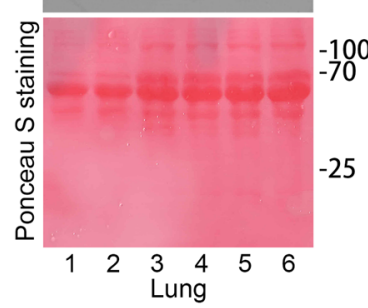

e

$f \rightarrow$ Mock $\mid$ Effector-infection

- Mock

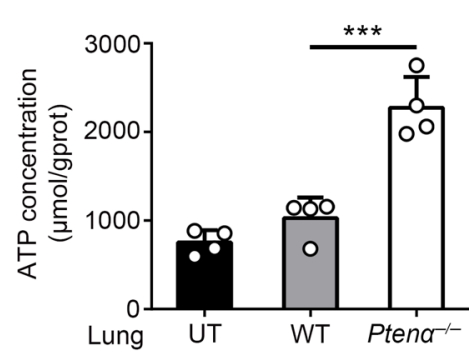

$\mathrm{h}$

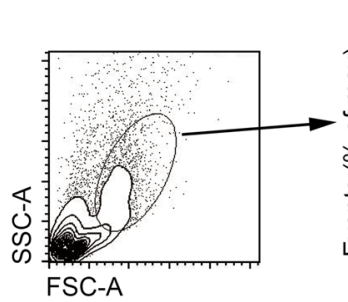

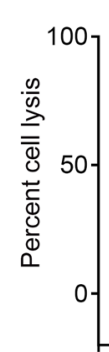

Effector-uninfection
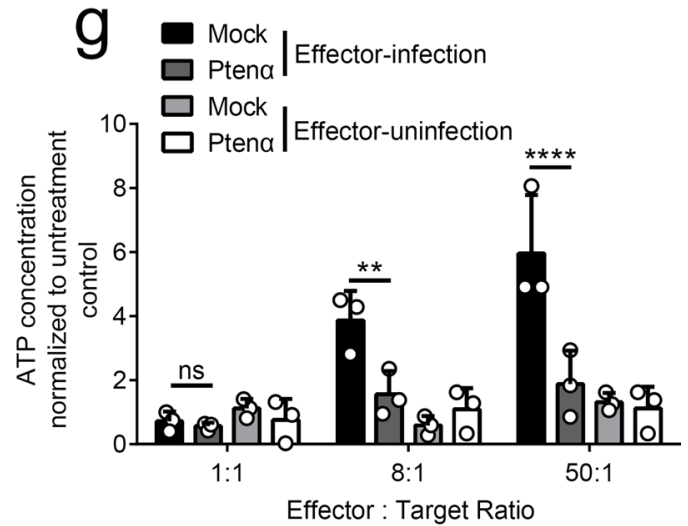

Effector : Target Ratio B16 Pten $^{--}$

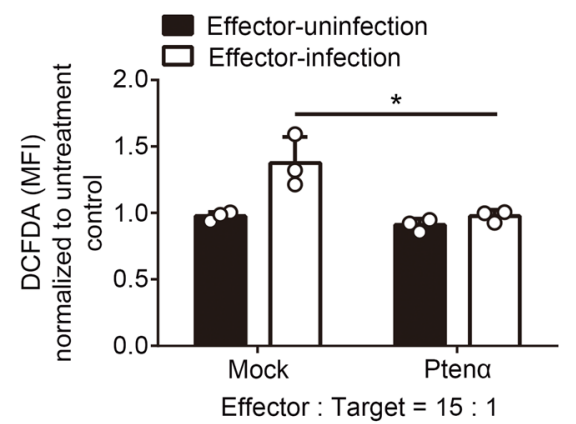

the effectiveness of tumor vaccination (Fig. 7i). Our data thus demonstrate that Gpx4 largely contributes to the stimulatory role of PTENa in cancer immune escape.
Collectively, we identify PTENa acts as phosphatase of eIF2 $\alpha$, which in turn promotes synthesis of peroxidases and blocks oxidative cell death, eventually ameliorating cancer immune evasion. 
Fig. 4 PTEN $\alpha$ promotes cell resistance to T cell cytotoxicity. a-e Wild-type and Pten $\alpha^{-/-}$mice were i.v. infected with $1 \times 10^{6}$ PFU LCMV-CI13. On day 7 post-infection, lungs were harvested from the mice. a The lungs were subjected to hematoxylin and eosin (H\&E) staining. The image is representative of four mice with similar results. Scale bar: $200 \mu \mathrm{m}$. b TUNEL staining of lungs from wild-type and Pten $\alpha^{-/-}$mice on day 7 post-infection. The image is representative of four mice with similar results. The red arrow indicates TUNEL positive cells. $\mathbf{c}$ The tissue interstitial fluids were isolated from the lungs and subjected to MS analysis. The differential proteins were analyzed using GSEA with GO gene sets. ES is an abbreviation of enrichment score, and NES represents normalized enrichment score. KO refers to Pten $\alpha^{-/-}$. Each sample is a mixture of fluids from three mice. $\mathbf{d}$ and $\mathbf{e}$ The interstitial fluids were isolated from the lungs, and used for immunoblot analysis with indicated antibodies (d), and ATP content assay (e) ( $n=4$ mixed samples from three mice, mean $\pm \mathrm{SD},{ }^{\star \star \star} P=0.0002$ ). For immunoblot analysis, equal amounts of loading proteins were showed by the Ponceau $\mathrm{S}$ staining. The relative ratios of Hsp70, Hsp90, and HMGB1 to Ponceau S-stained total proteins were indicated. UT, untreatment. f-h In vitro cytotoxicity assay of B16 cells. Mock or PTEN $\alpha$ expressing Pten ${ }^{-/-}$B16 cells pulsed with $\mathrm{GP}_{33-41}$ peptide were used as target cells. Effector cells and target cells were incubated at indicated ratios. Effector-uninfection refers to $T$ cells from uninfected mice and acts as a negative control. $\mathbf{f} 20 \mathrm{~h}$ post-incubation, B16 cells were washed to remove lymphocytes and counted using CCK-8 $\left(n=3\right.$ cell cultures, mean \pm SD, $\left.{ }^{\star \star \star} P(2)=0.0006,{ }^{\star \star \star} P(25)=0.0003,{ }^{\star \star \star \star} P<0.0001\right)$. g Culture medium was collected $20 \mathrm{~h}$ post-incubation and centrifuged to remove cells and debris. The supernatant was subjected to ATP content assay ( $n=3$ cell cultures, mean $\pm \mathrm{SD}, \mathrm{ns}$, not significant, $\left.{ }^{\star \star} P=0.0078,{ }^{\star \star \star \star} P<0.0001\right)$. Untreatment control refers to pulsed B16 cells without effector incubation. $\mathbf{h} 12 \mathrm{~h}$ postincubation, B16 cells were harvested and stained with DCFDA, using for flow cytometry analysis. Cells with higher intensity of FSC and SSC were gated and considered as B16 cells. Mean fluorescence intensity (MFI) of DCFDA was used for statistical analysis ( $n=3$ cell cultures, mean \pm SD, ${ }^{\star} P=0.0255$ ). Untreatment control refers to pulsed B16 cells without effector incubation. Statistical significance was assessed by two-tailed unpaired Student's $t$ test (h) or one (e) or two ( $\mathbf{f}$ and $\mathbf{g}$ )-way ANOVA followed by Tukey's multiple comparisons test. Data are representative of two (a, b, d-h) independent experiments. Source data are provided as a Source Data file. See also Supplementary Fig. 7.

\section{Discussion}

Despite the wide application of immunotherapy in the treatment of cancers, only a subset of patients responds to the treatment, and the clinical efficacy is often compromised along with tumor development ${ }^{10}$. Notably, a recent study uncovers that PTEN mutations are frequently observed in immune-resistant cancers ${ }^{25}$. Although the combination of immunotherapies and PI3K-AKT pathway inhibitors elicits partially effective to reinvigorate tumorinfiltrating $\mathrm{T}$ cells ${ }^{26}$, the immunosuppressive tumor environment can hardly be transformed, which suggests that other signaling pathways are also triggered in addition to PTEN dysfunction. Here we identify that presence of PTENa in PTEN-mutant cancers counteracts immune attack and promotes tumor immune escape (Supplementary Fig. $10 \mathrm{~g}$ ). Rather than affecting $\mathrm{T}$ cell priming, tumor-intrinsic PTENa restricts T cell-mediated cytotoxicity through limiting ferroptosis, which attenuates immunogenic cell death and maintains immunosuppressive niche. Our data thus indicate that PTENa can be a potential target of immune therapy to regulate the susceptibility of tumor cells to immune responses.

As an N-terminally extended isoform of PTEN, PTENa translates through a CUG codon upstream of and in-frame with the coding region of canonical PTEN ${ }^{15}$. Accompanied with PTEN, the phosphatase-inactive mutations also impair the phosphatase activity of PTENa. However, we find that the stopgained mutations, which result in PTEN instability and degradation, hardly affect PTENa stabilization. Notably, the presence of stop-gained mutations of PTEN is correlated with less T-cell infiltration and worse patient outcome, suggesting the oncogenic role of PTENa in tumor development. Interestingly, the role of PTENa in tumors seems complicated due to the opposite effects of PTENa on tumor growth in glioblastoma and liver cancer ${ }^{14,16}$. Although clinical analysis reveals that sustained expression of PTENa in liver cancer tissue is correlated with accelerated tumor progression, both types of research use the model of nude mice bearing tumor xenograft and fail to investigate the role of PTENa in anti-tumor immunity ${ }^{14,16}$. Herein, we identify the immunosuppressive function of PTENa, which attenuates cancer cell susceptibility to $\mathrm{T}$ cell-mediated cytotoxicity through limiting oxidative cell death, thereby promoting cancer escape from immune eradication.

Among the inhibitory effects of PTENa on host antitumor immune response, we regard its negative role in oxidative cell death as the core mechanism in vivo. First, the tumor vaccination model reveals that tumor-intrinsic rather than $\mathrm{T}$ cell-intrinsic PTENa mainly contributes to cancer immune evasion. Second, both in vitro killing assay and $\mathrm{T}$ cell adoptive transfer model demonstrate that reduction of immunogenic cell death and ensued DAMPs release is the cause of limited recruitment of immune cell. Third, chronic viral infection and BMT models demonstrate that reduced cell death and consequently persistent antigenic stimulation induced by PTENa drive exhausted T cell formation. Above all, our results demonstrated that, through protecting tumor cells from $\mathrm{T}$ cell killing, PTENa limits the infiltration of leukocytes in tumors and impairs $\mathrm{T}$ cell effector function, eventually leading to tumor immune escape.

The physiological function of PTENa remains largely unknown. Here we demonstrate that, as a phosphatase of eIF2 $\alpha$, PTENa maintains host protein synthesis, and limits stress granule formation. Stress granules have a great impact on cells' adaption to environmental changes through selective modulation of host protein synthesis under various stress conditions ${ }^{23,24}$. We found that PTENa restricts stress granule formation and maintains translation of proteins associated with oxidation-reduction processes, which in turn limits oxidative cell death and ameliorates the inflammatory response by blocking DAMPs release. In accordance with viral infection, it has been reported that Pseudomonas aeruginosa infection triggers severe immunopathology in mice without PTEN-L (PTENa) ${ }^{27}$. Our work thus supports the notion that the presence of PTENa is critical for host protection from pathogen-induced tissue damage. In PTEN mutant cancer, this process is hijacked, thereby promoting the adaption of tumor cells to $\mathrm{T}$ cell cytotoxicity, resulting in tumor immune escape.

Ferroptosis has been implicated in a variety of pathological disorders, such as neurodegenerative events, ischemia/reperfusion injury, and bacterial infection ${ }^{28}$. Besides, a previous study has revealed that CTLs can kill cancer cells by inducing ferroptotic cell death ${ }^{12}$. A recent study also uncovers that the sensitivity of tumor cells to oxidative stress and ferroptosis limits their metastasis through blood ${ }^{29}$. In this study, our results identify the inhibitory role of PTENa in the modulation of ferroptosis. Through neutralizing intracellular ROS, PTENa enhanced cancer cell resistance against $\mathrm{T}$ cell killing. Moreover, the presence of PTENa promoted cancer metastasis through blood. Therefore, these results further confirm the importance of PTENa in cancer immune escape and highlight the potential of targeting PTENa to be a promising way in cancer treatment. 


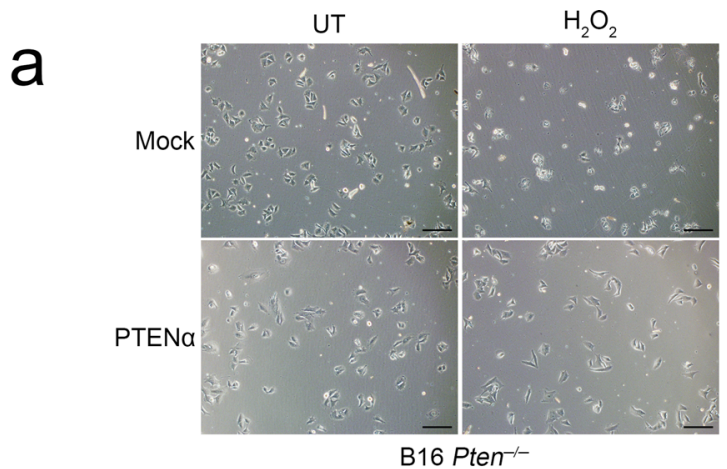

b
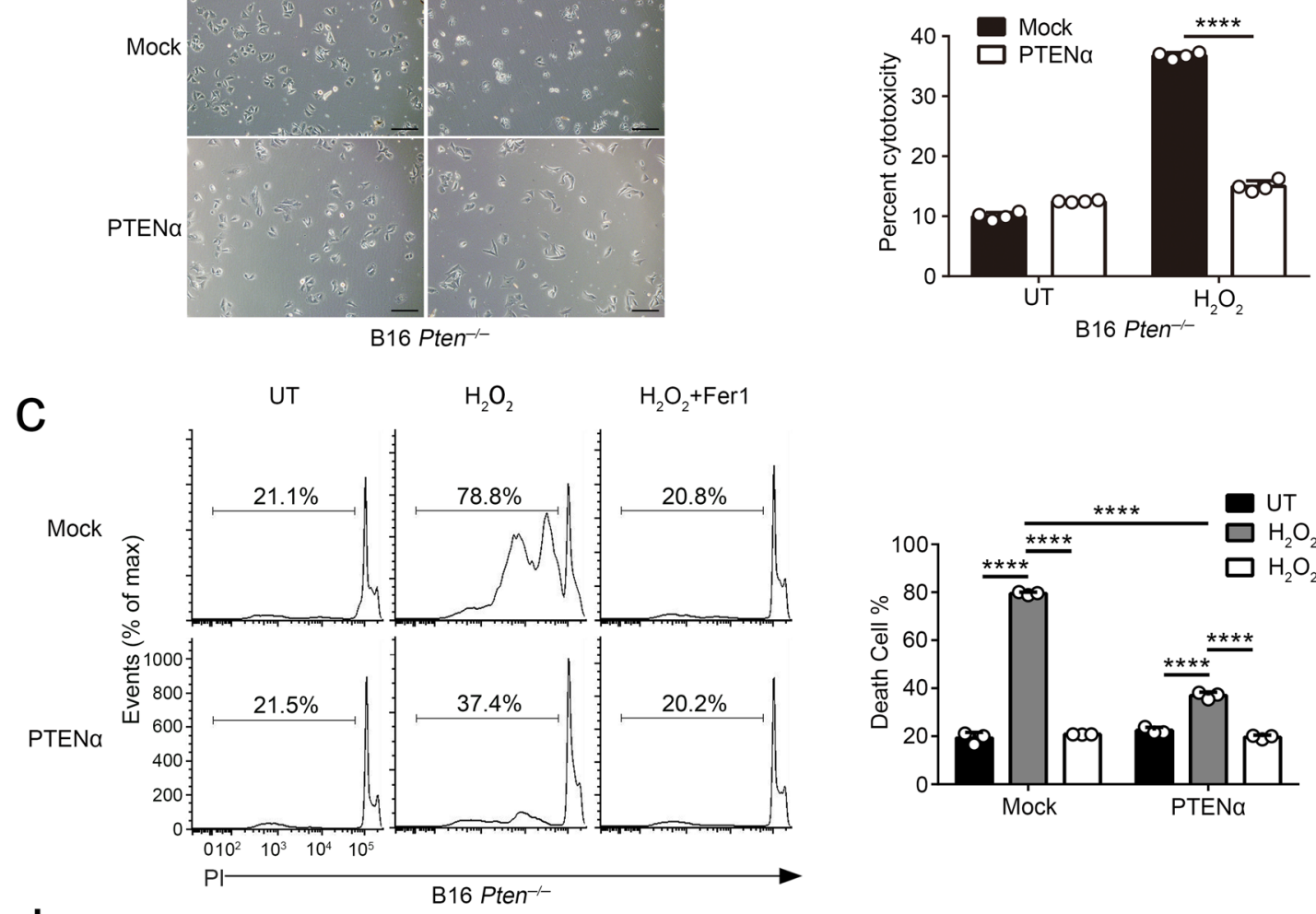

d
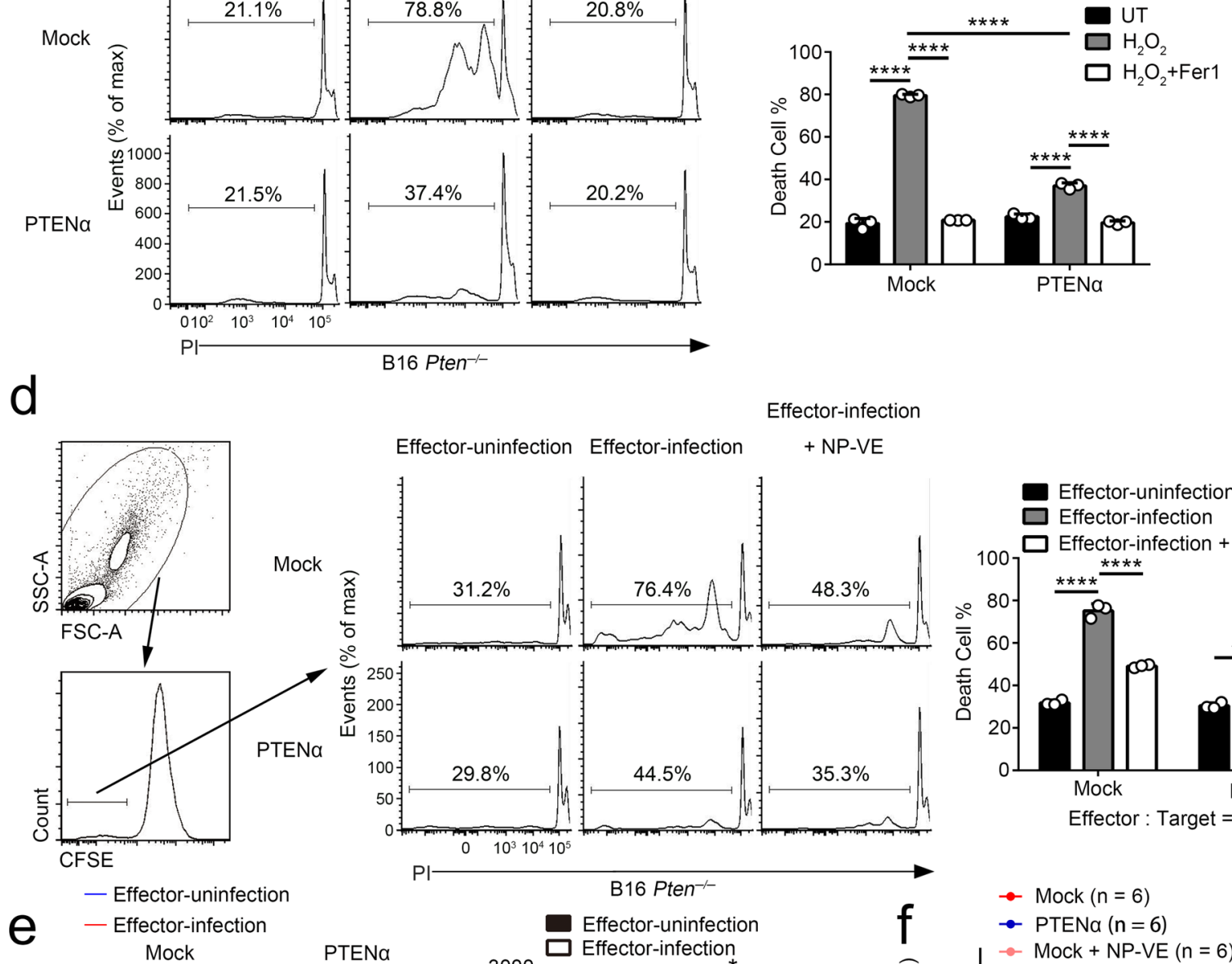

Effector-infection

+ NP-VE

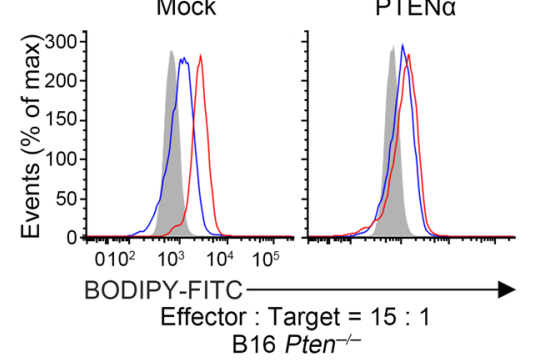

Effector-uninfection Effector-infection

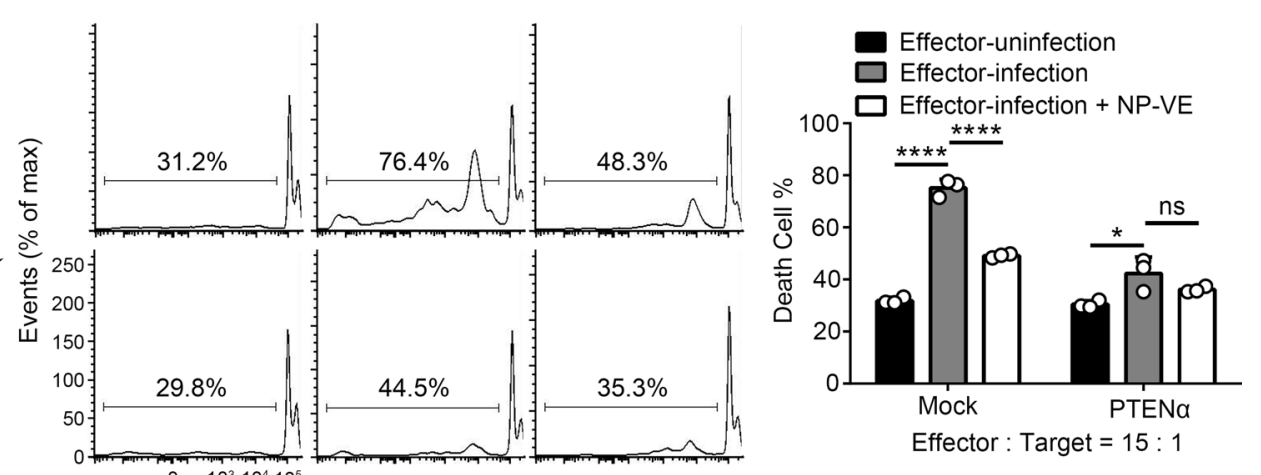

\section{Methods}

Mice. Pten $\alpha^{-/-}$mice (C57BL/6J background) were generated and reported in our previous study ${ }^{30}$. Balb/c and Balb/c nude mice were purchased from Charles River Laboratories. 6-8 weeks old male mice were used for the study. All animals were maintained under a specific pathogen-free condition at the Department of

Laboratory Animal Science of Peking University Health Science Center and reared in standard conditions with controlled temperature $\left(20-26^{\circ} \mathrm{C}\right)$, humidity $(40-70 \%)$, and 12/12-hour dark/light cycle. The animal experimental protocols were approved by the Ethics Committee of Peking University Health Science Center, and the animal testing and research comply with all relevant ethical regulations.

Cells. B16-F10, HEK-293T, Molt4, Jurkat, CT26, and HeLa cells were from American Type Culture Collection (ATCC). MEFs were obtained through mincing 
Fig. 5 PTEN $\alpha$ restricts oxidative cell death. a Microscopy analysis of $\mathrm{H}_{2} \mathrm{O}_{2}(500 \mu M)$ treated Mock or PTEN $\alpha$-expressing B16-Pten ${ }^{-/-}$cells. UT untreatment. Scale bar: $100 \mu \mathrm{m}$. b Mock or PTEN $\alpha$-expressing B16-Pten ${ }^{-/-}$cells were treated with $500 \mu \mathrm{M} \mathrm{H}_{2} \mathrm{O}_{2}$ and the percent cytotoxicity was assessed using $\mathrm{LDH}$ release assay $\left(n=4\right.$ cell cultures, mean $\pm \mathrm{SD}$, $\left.{ }^{\star \star \star \star} P<0.0001\right)$. UT untreatment. c Mock or PTEN $\alpha$-expressing B16-Pten ${ }^{-/-}$cells were treated with $500 \mu \mathrm{M} \mathrm{H}_{2} \mathrm{O}_{2}$ in the absence or presence of $1 \mu \mathrm{M}$ Fer1. Cells were stained with propidium iodide (PI), followed by flow cytometric analysis. All cells were gated. The death cell rates were used for statistical analysis $\left(n=3\right.$ cell cultures, mean $\left.\pm \mathrm{SD},{ }^{\star \star \star \star} P<0.0001\right)$. UT untreatment. $\mathbf{d}$ and $\mathbf{e}$ In vitro cytotoxicity assay for B16 cells. Mock or PTEN $\alpha$-expressing B16-Pten ${ }^{-/-}$cells pulsed with $\mathrm{GP}_{33-41}$ peptide were used as target cells. Effector cells and target cells were incubated at indicated ratios. Effector-uninfection refers to non-antigen-specific $T$ cells and acts as a negative control. $\mathbf{d}$ Before incubation, $\mathrm{CD}^{+}{ }^{+} \mathrm{T}$ cells were stained with CFSE, then the effector cells and target cells were incubated in the presence or absence of $20 \mathrm{mM}$ NP-VE. $20 \mathrm{~h}$ postincubation, the cells were harvested and subjected to PI staining. Death cells in the CFSE ${ }^{-}$cells were assessed by flow cytometric analysis ( $n=3$ cell cultures, mean $\pm S D$, ns not significant, ${ }^{\star} P=0.0188$, $\left.{ }^{\star \star \star \star} P<0.0001\right)$. Gating strategies were shown. $\mathbf{e} 12 \mathrm{~h}$ post-incubation, cells were harvested and stained with BODIPY-C11, followed by flow cytometric analysis. The gating strategy was identical to that in Fig. $4 \mathrm{~h}$. MFI of the target cells was used for statistical analysis ( $n=3$ cell cultures, mean \pm SD, ${ }^{\star} P=0.0207$ ). f Mock or PTEN $\alpha$ transfected Pten ${ }^{-/}-$B16 cells were subjected to the cancer vaccination model and subcutaneously injected in the presence or absence of $50 \mu \mathrm{l}$ of $5 \mathrm{M} \mathrm{NP-VE}$. Tumor volumes were monitored over time, and the statistical significances between the Mock groups were indicated (PTEN $\alpha+N P-V E, n=7$ mice; other groups, $n=6$ mice, mean \pm s.e.m., ${ }^{\star} P=0.0465,{ }^{\star \star} P$ $\left.(\mathrm{d} 20)=0.0015,{ }^{\star \star} P(\mathrm{~d} 20)=0.0019\right)$. Statistical significance was assessed by two-tailed unpaired Student's $t$ test $(\mathbf{b}, \mathbf{e}, \mathbf{f})$ or one (d) or two (c)-way ANOVA followed by Tukey's multiple comparisons test. Data are representative of two (a-f) independent experiments. Source data are provided as a Source Data file. See also Supplementary Figs. 8 and 9.

13.5-day embryos, digesting with trypsin, and filtering through a $70 \mu \mathrm{m}$ strainer. For the construction of B16-Pten ${ }^{-1-}$ cell lines, the CRISPR-Cas9 system was used. In brief, the guide sequence "TGTGCATATTTATTGCATCG" targeting Pten was cloning into the PX330 plasmid. The PX330 plasmid was co-expressed with Cas9 in the B16 cells and selected with G418. The cells were seeded in a 96-well plate at the density of 50 cells per well. After 2-3 weeks of expansion, the clones were harvested and examined with sequencing and western blot.

Antibodies and reagents. Following commercial antibodies were used in this study. clone number, catalog number and dilutions were shown in turn: anti-PTEN (138G6, 9559, 1:1000), anti-eIF2 $\alpha$ (D7D3, 5324, 1:1000) and antibody to phosphorylated eIF2a (Ser51) (119A11, 3597, 1:1000) (all from CST); anti-GAPDH (1C4, KM9002T, 1:5000) and anti-GFP (9F6, KM8009, 1:5000) (both from Sungenebiotech); anti-FLAG (M2, F3165, 1:5000, Sigma-Aldrich); anti- $\alpha$-tubulin (2F9, M175-3, 1:5000), H-2D ${ }^{\mathrm{b}}$ LCMV gp33 Tetramer-KAVYNFATC (TB-5002-1, 1:250) (both from MBL); anti-puromycin (17H1, MABE341, 1:1000, merck-millipore); anti-GPX4 (E-12, sc-166570, 1:1000) and anti-LCMV (M104, sc-57894, 1:100) (all from Santa Cruz); anti-Hsp90 (OTI4C10, TA-12, 1:1000, ZSGB-BIO); anti-NPTEN (EPR23729-4, ab260011, 1:1000), anti-HMGB1 (EPR3507, ab79823, 1:1000) and anti-Hsp70 (EPR16892, ab181606, 1:1000) (all from abcam); anti-IL-17A (eBiol7B7, 12-7177-81, 1:250), anti-IL-10 (JES5-16E3, 12-7101-41, 1:250), antiFoxp3 (FJK-16s, 17-5773-82, 1:250), anti-PD-1 (J43, 46-9985-82, 1:250), anti-TIM3 (RMT3-23, 25-5870-82, 1:250), anti-LAG3 (C9B7W, 17-2231-82, 1:250), antiCD45 (30-F11, 25-0451-81, 1:250), anti-CD25 (PC61.5, 12-0251-83, 1:250), antibody to IFN- $\gamma$ (XMG1.2, 17-7311-82, 1:250) and antibody to TNF- $\alpha$ (MP6-XT22, 25-7321-82, 1:250) (all from eBioscience); Anti-CD4 (GK1.5, 35-0041-U500, 1:250) and anti-CD8 (53-6.7, 35-0081-U500 and 20-0081-U100, 1:250) (both from Tonbo); anti-G3BP1 (A14836, 1:1000, ABclonal); Anti-CD3e (145-2C11, 100301, $2 \mu \mathrm{g} / \mathrm{ml})$, anti-CD28 (37.51, 102101, $1 \mu \mathrm{g} / \mathrm{ml})$, anti-IL-4 (11B11, 504101, $10 \mu \mathrm{g} / \mathrm{ml}$ ) and anti-IFN $\gamma$ (XMG1.2, 505801, $10 \mu \mathrm{g} / \mathrm{ml}$ ) (all from BioLegend).

The reagents used in this study were as follow z-VAD-Fmk, Erastin, RSL3 and ferrostatin-1 (Fer-1), Selleck; $\mathrm{H}_{2} \mathrm{O}_{2}$, Cycloheximide (CHX), propidium iodide (PI), N-Acetyl-L-cysteine (NAC), Deferoxamine (DFO), and $\alpha$-tocopherol ( $\alpha$-Toc) Sigma; H2DCFDA, GeneCopoeia; Thapsigargin (TG) and Necrostatin-1 (Nec-1), abcam; L- ${ }^{35}$ S-Methionine, PerkinElmer; puromycin, ACROS; Recombinant mouse TNF- $\alpha$, Biolegend; Annexin V and 7-AAD, ebioscience; CFSE, Tonbo; BODIPYC11, Invitrogen.

Short hairpin RNA infection assay. pLKO.1-puro was used to knock down gene expression. Sequence for Gpx4 shRNA interference was 5'-CCGGGGAGCCCAT TCCTGAACCTTTCTCGAGAAAGGTTCAGGAATGGGCTCCTTTTTG- ${ }^{\prime}$, and sequence for Pten shRNA interference was $5^{\prime}$ - CCGGGGGTAAATACGTTCTT CATACCTCGAGGTATGAAGAACGTATTTACCCTTTTTG-3'

Cell proliferation assay. Different numbers of cells were seeded into the 96-well plates by the gradient. Then the cells were subjected to a Cell counting Kit-8 (CCK8 , Dojindo) assay following the manufacturer's instructions to draw the stand curve. For assessment of cell proliferation, the B16 cells were seeded into a 96-well plate at the density of 1000 cells per well in $100 \mu \mathrm{L}$ DMEM medium containing $10 \%$ (vol/vol) fetal bovine serum (FBS). The cells were cultured at $37^{\circ} \mathrm{C}$ and measured using CCK- 8 daily. The cell numbers were determined by comparing them with the stand curve.

Colony formation assay. B16 cells were seeded in a six-well plate at the density of 100 cells per well and cultured at $37^{\circ} \mathrm{C}$ for 7 days. After fixing in methanol for 20 min and washing with PBS twice, the cells were stained with Crystal Violet
Staining Solution (Beyotime) at room temperature for $20 \mathrm{~min}$, using for photographing.

Melanoma mouse model. For the cancer vaccination model, $1 \times 10^{5} \mathrm{~B} 16-\mathrm{F} 10$ cells were irradiated ( $40 \mathrm{~Gy}$ ), and cultured at $37^{\circ} \mathrm{C}$ overnight. Then the dying cells were collected and injected subcutaneously into the left flank of C57BL/6 mice. 10 days post-injection, $1 \times 10^{6} \mathrm{~B} 16$ cells were re-injected into the right flank of the mice through subcutaneous injection. The tumor volumes were monitored over time.

For adoptive T cell transfer assay, B16 cells were infected with 1 MOI LCMV-Cl13 for $24 \mathrm{~h}$, and subcutaneous injected into the right flank of Balb/c nude mice. Meanwhile, C57BL/6 mice were intravenous (i.v.) infected with $1 \times 10^{6}$ LCMV-Cl13, and CD8 ${ }^{+}$ $\mathrm{T}$ cells were isolated from the spleen of the mice on day 7 post infection. Then the $\mathrm{CD}^{+} \mathrm{T}$ cells were transferred into the tumor-bearing nude mice on day 10,14 , and 18 post tumor inoculation. Tumor volumes were monitored overtime.

Viral infection. For LCMV chronic infection mice model, 6-8-week-old mice were infected by i.v. injection with indicated titers of LCMV-Cl13.

Quantitative real-time PCR (qRT-PCR). TRIzol reagents (invitrogen) were used to isolate total RNA from cells or tissues, followed by reverse transcribing with GoScriptTM Reverse Transcription System (Promega). TransStart Top Green qPCRSuperMix (TransGen Biotech) was used for real-time PCR. qRT-PCR was performed using Applied Biosystems 7500 Fast \& 7500 Real-Time PCR System and 7500 Software v2.3 (Applied Biosystems) (all primers are listed in Supplementary Table 2).

Bone marrow transplantation (BMT). To obtain bone marrow cells, donor mice were sacrificed, and the femurs and tibias were harvested. The bone marrow canals were washed out with RPMI1640 medium containing 1\% (vol/vol) FBS, and the bone marrow cells were collected, followed by red blood cell (RBC) lysis. Recipient mice were lethally irradiated ( $896 \mathrm{cGy} /$ mouse), and i.v. injected with $5 \times 10^{6}$ bone marrow cells. Experiments on transplanted mice were performed after a latency of 30 days to ensure bone marrow reconstitution.

In vitro cytotoxicity assay. C57BL/6 mice were untreated or i.v. infected with $1 \times 10^{6} \mathrm{PFU}$ LCMV-Cl13. On day 7 post infection, lymphocytes were isolated from spleens of the infected mice, and the $\mathrm{CD}^{+} \mathrm{T}$ cells were isolated using MagniSort ${ }^{\mathrm{TM}}$ Mouse CD8 Positive Selection Kit (eBioscience). The CD8 ${ }^{+} \mathrm{T}$ cells were incubated with target cells pulsed with $2 \mu \mathrm{g} / \mathrm{ml} \mathrm{GP} \mathrm{m3}_{31}$ peptide at indicated ratios. $8 \mathrm{~h}$ post incubation, target cells were collected and stained with DCFDA, using for flow cytometry analysis. For percent cell lysis rate, target cells were washed to remove lymphocytes, and live cells were measured using Cell counting Kit-8 (CCK8 , Dojindo) following the manufacturer's instructions.

Detection of ROS production. ROS-sensitive fluorescent probe $2^{\prime}, 7^{\prime}$-dichlorodihydrofluorescein diacetate (H2DCFDA) was used to detect generation of ROS in cultured cells. Briefly, cells were washed with PBS, followed by staining with $100 \mathrm{mM}$ DCFDA for $30 \mathrm{~min}$ at $37^{\circ} \mathrm{C}$. After washing with FACS (PBS + 1\% (vol/ vol) FBS), stained cells were subjected to flow cytometry analysis.

For detection of lipid peroxidation, cells were collected, and stained with $2 \mu \mathrm{M}$ BODIPY-C11. Lipid ROS level was determined by flow cytometry analysis with excitation at $488 \mathrm{~nm}$. 

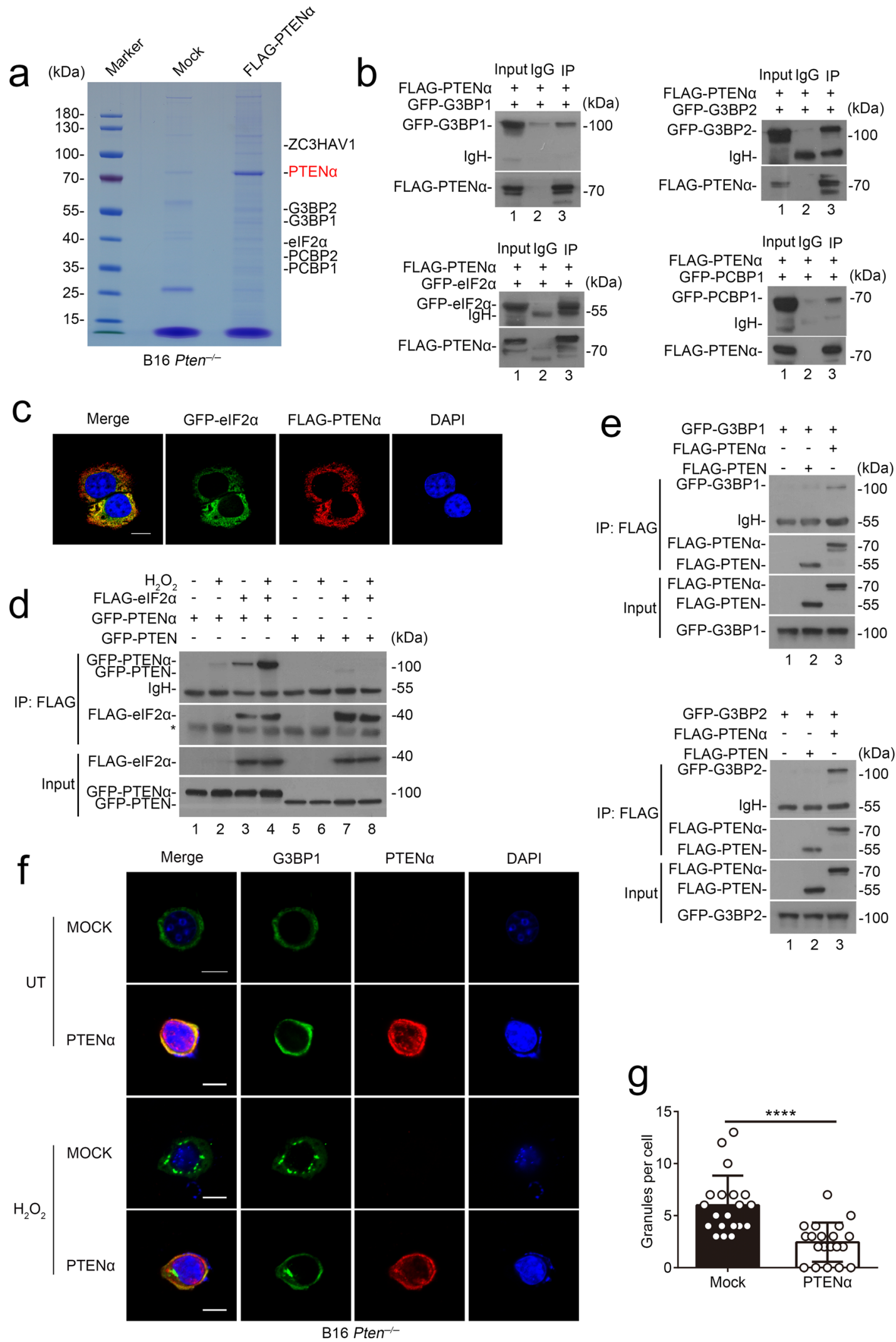

\section{9}

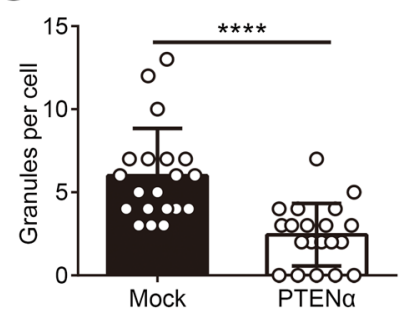

TUNEL staining. For the detection of Terminal deoxynucleotidyl transferase (TdT)-mediated dUTP nick end labeling (TUNEL) positive cells, the in-situ cell death detection kit (Roche) was used according to the manufacturer's instructions.
Confocal microscopy. Cells were seeded on cover glasses. $12 \mathrm{~h}$ later, cells were fixed with $100 \%$ acetone, permeabilized with $0.5 \%$ (vol/vol) Triton X-100 and then blocked using 1\% (wt/vol) BSA dissolved in PBS. Primary antibody was applied for at least $1 \mathrm{~h}$, 
Fig. 6 PTEN $\alpha$ blocks stress granules formation during oxidative stress. a MS analysis of PTEN $\alpha$ associated proteins by FLAG pull-down assay in Pten ${ }^{-/-}$ B16 cells treated with $100 \mu \mathrm{M} \mathrm{H}_{2} \mathrm{O}_{2}$. Proteins that interact with PTEN $\alpha$ are indicated on the right (outlined text). b HEK293T cells were co-transfected with FLAG-tagged PTEN $\alpha$ and GFP-tagged indicated vectors, followed by $100 \mu \mathrm{M} \mathrm{H}_{2} \mathrm{O}_{2}$ treatment for $3 \mathrm{~h}$. Cell lysates were immunoprecipitated with anti-FLAG antibodies or normal mouse IgG. The immunoprecipitated proteins were subjected to immunoblot analysis with anti-GFP antibodies. c HeLa cells were cotransfected with FLAG-tagged PTEN $\alpha$ and GFP-tagged elF2 $\alpha$ vectors, and interaction between the proteins was shown by confocal fluorescence microscopy. DAPI was used to indicate nuclear. Scale bars, $10 \mu \mathrm{m}$. d HEK293T cells were co-transfected with FLAG-tagged elF2 $\alpha$ and GFP-tagged PTEN or PTEN $\alpha$ vectors in the absence or presence of $\mathrm{H}_{2} \mathrm{O}_{2}(100 \mu \mathrm{M})$. Cell lysates were immunoprecipitated with anti-FLAG antibody and analyzed by immunoblot with anti-GFP antibody. " ${ }^{\star \prime}$ refers to unspecific band. e HEK293T cells were transfected to express FLAG-tagged PTEN or PTEN $\alpha$, together with GFP-tagged G3BPs, followed by treatment of $100 \mu \mathrm{M} \mathrm{H}_{2} \mathrm{O}_{2}$ for $3 \mathrm{~h}$. Cell lysates were immunoprecipitated with anti-FLAG antibody and assessed by immunoblot analysis with anti-GFP antibody. $\mathbf{f}$ and $\mathbf{g}$ Pten ${ }^{-/-}$B16 cells were transfected with Mock or FLAG-tagged PTEN $\alpha$ vectors, followed by confocal fluorescence microscopy analysis in the presence or absence of $100 \mu \mathrm{M} \mathrm{H}_{2} \mathrm{O}_{2}$ with anti-G3BP1 and anti-PTEN (138G6) antibodies (f). Granules in the cells were counted, using for statistical analysis $(\mathbf{g})\left(n=20\right.$ cells, mean $\left.\pm \mathrm{SD},{ }^{\star \star \star \star} P<0.0001\right)$. Scale bars, $10 \mu \mathrm{m}$. UT, untreatment. Statistical significance was assessed by a two-tailed unpaired Student's $t$ test $(\mathbf{g})$. Data are representative of two $(\mathbf{b}-\mathbf{i})$ independent experiments. Source data are provided as a Source Data file.

followed by incubation with fluorophore-conjugated secondary antibody for $1 \mathrm{~h}$. DAPI (BioDee Biotechnology) was used to indicate nuclear, and cells were evaluated with fluorescence microscopy using NIS-Elements AR Analysis 4.20.00 64 bit software (Nikon). NIS Elements Viewer 4.20 was used to analyze the confocal microscopy data.

LDH release assay. The CytoTox 96R Non-Radioactive Cytotoxicity Assay (Promega) was used to detect lactate dehydrogenase (LDH) release and cell death rates. In brief, cells were seeded in 96-well plates and treated with indicated reagents. The experimental LDH release and maximum LDH release were determined according to the manufacturer's instructions, and the percentage of cytotoxicity was calculated by dividing experimental LDH release by maximum LDH release.

Interstitial fluid isolation. Tissue was harvested from mice. Bulk tissue was placed on a $40 \mu \mathrm{m}$ cell strainer, followed by centrifuging at $45 \times \mathrm{g}$ for $5 \mathrm{~min}$ at $4^{\circ} \mathrm{C}$ to remove surface fluid. Then the tissue was centrifuged at $500 \times g$ for $10 \mathrm{~min}$ at $4{ }^{\circ} \mathrm{C}$, and the interstitial fluid was collected. After spinning at $10,000 \times g$ for $10 \mathrm{~min}$ at $4{ }^{\circ} \mathrm{C}$ to remove insoluble particles, the supernatant was subjected to immunoblot analysis and ATP content assay.

Ponceau S staining. Protein samples were subjected to immunoblot analysis. After the electrophoresis and transmembrane, polyvinylidene fluoride membrane was stained by Ponceau S staining solution (Beyotime) for $30 \mathrm{~min}$. The membrane was washed and used for scanning.

ATP content assay. ATP contents in the interstitial fluids were detected using ATP assay kit (Nanjing Jiancheng) according to the manufacturer's instructions.

Propidiumiodide (PI) staining. Cultured cells were digested and fixed with $100 \%$ Ethanol, followed by staining with $1.1 \mathrm{mg} / \mathrm{ml}$ PI containing $1 \%$ (vol/vol) TritonX100. Stained cells were used for flow cytometry analysis. For cell cycle analysis, peaks of cells with diploid DNA content were analyzed. Determination of cell death was performed by analyzing the sub-G1 peak.

Hematoxylin and eosin (H\&E) staining. Tissue specimens were fixed in 10\% (vol/ vol) neutral buffered formalin followed by paraffin-sectioning and H\&E staining.

Preparation of lymphocytes. Lymphocytes in spleen were isolated by grinding the tissues in PBS containing 1\% (vol/vol) FBS, followed by filtering through a $75 \mu \mathrm{m}$ strainer. To isolate lymphocytes infiltrating in the lung, minced tissues were digested with $0.5 \mathrm{mg} / \mathrm{ml}$ Collagenase D (Roche) and $25 \mu \mathrm{g} / \mathrm{ml}$ DNase I (Sigma) at $37^{\circ} \mathrm{C}$ for $30 \mathrm{~min}$. Then the tissues were grinded, and filtered through a $75 \mu \mathrm{m}$ strainer. A $40 \%(10 \mathrm{ml}) / 70 \%(5 \mathrm{ml})$ Percoll gradient (GE Healthcare) was used to isolate the tissues by centrifuging $800 \times g$ for $20 \mathrm{~min}$. Cells at the inter-layer were collected and counted for further operation. For isolation of TIL, tumors were minced, grinded and filtered just like the lungs mentioned above. Then the cells were resuspended in $5 \mathrm{ml}$ FACS buffer, adding to a $40 \%(5 \mathrm{ml}) / 80 \%(5 \mathrm{ml})$ Percoll gradient. The gradient was centrifuged at $400 \times g$ for $45 \mathrm{~min}$, and the cells at the inter-layer of $40 \% / 80 \%$ Percoll gradient was collected for further operation.

In vitro $\mathbf{T}$ cell differentiation assay. Naïve $\mathrm{CD} 4^{+} \mathrm{T}\left(\mathrm{CD} 4^{+} \mathrm{CD} 44^{\text {low }}\right)$ cells from spleens of C57BL/6 mice were sorted, and activated with plate-bound $2 \mu \mathrm{g} / \mathrm{ml}$ antiCD3 (145-2C11; BioLegend) and $1 \mu \mathrm{g} / \mathrm{ml}$ anti-CD28 (37.51; BioLegend) antibodies. The cells were treated with various cytokines or antibodies for polarization. For Th1 cell differentiation, $10 \mu \mathrm{g} / \mathrm{ml}$ anti-IL-4 (11B11; BioLegend) antibody and $10 \mathrm{ng} / \mathrm{ml} \mathrm{IL-12}$ (R\&D) were used. For Th17 polarization, $20 \mathrm{ng} / \mathrm{ml}$ IL-6 (R\&D),
$5 \mathrm{ng} / \mathrm{ml}$ TGF- $\beta$ (R\&D), $10 \mu \mathrm{g} / \mathrm{ml}$ anti-IFN- $\gamma$ (XMG1.2; BioLegend) and $10 \mu \mathrm{g} / \mathrm{ml}$ anti-IL-4 antibodies were used. For $\operatorname{Tr} 1$ cell differentiation, cells were treated with $50 \mathrm{ng} / \mathrm{ml} \mathrm{IL-27} \mathrm{(eBioscience),} 10 \mu \mathrm{g} / \mathrm{ml}$ anti-IFN- $\gamma$ and $10 \mu \mathrm{g} / \mathrm{ml}$ anti-IL-4 antibodies. For iTreg polarization, $1 \mathrm{ng} / \mathrm{ml}$ TGF- $\beta, 4 \mathrm{ng} / \mathrm{ml}$ IL-2 (R\&D), $10 \mu \mathrm{g} / \mathrm{ml}$ antiIFN- $\gamma$ and $10 \mu \mathrm{g} / \mathrm{ml}$ anti-IL- 4 antibodies were used. The cells were cultured at $37^{\circ} \mathrm{C}$ for 48 or $72 \mathrm{~h}$, followed by flow cytometry analysis.

For detection of activation induced cell death, Naïve $\mathrm{CD} 4^{+} \mathrm{T}$ cells were activated with plate-bound $2 \mu \mathrm{g} / \mathrm{ml}$ anti-CD3 and $1 \mu \mathrm{g} / \mathrm{ml}$ anti-CD28 antibodies. $72 \mathrm{~h}$ later, cells were collected, and subjected to Annexin V/7-AAD staining.

CFSE staining. For CFSE staining, splenocytes were harvested, and washed twice with PBS, followed by staining with CFSE at dark for $10 \mathrm{~min}$. Then the cells were washed twice with PBS containing 5\% (w/w) FBS, and subjected to flow cytometry analysis.

Annexin V/7-AAD staining. Annexin V/7-AAD staining was performed using Annexin V Apoptosis Detection Kit (eBioscience), following the manufacturer's instructions.

Flow cytometry. For surface staining, cells were incubated with specific antibodies for $30 \mathrm{~min}$ at room temperature. The flow cytometry analyzer (BD Biosciences), FACSuite Software Bundle v1.0 (BD Biosciences), and FACSDiva Software v6.1 (BD Biosciences) were used for acquiring and analyzing the cells. The FACS data were analyzed with FlowJo v7.6.1 software. To perform intracellular cytokine staining, cells were stimulated with $100 \mathrm{ng} / \mathrm{ml}$ PMA and $500 \mathrm{ng} / \mathrm{ml}$ ionomycin, or $2 \mu \mathrm{g} / \mathrm{ml} \mathrm{GP}{ }_{33-41}$ peptide together with GolgiPlug and GolgiStop (BD) for $5 \mathrm{~h}$. Subsequently, cells were fixed and permeabilized, followed by staining with specific anti-cytokine antibodies.

Pulldown assay and mass spectrometry. H1299 cells were seeded and transfected with FLAG-tagged-PTENa. The whole-cell extracts were incubated with FLAG-beads (Sigma-Aldrich), followed by washing four times using PBS-N (PBS with $0.1 \%$ (vol/vol) NP40). Immunoprecipitated proteins were eluted using the $3 \times$ FLAG-peptide and subjected to SDS-PAGE. After staining with Coomassie Brilliant blue, the gel was excised and subjected to in-gel trypsin digestion and dried. $10 \mu \mathrm{l} 0.1 \%$ formic acid was used to dissolve the peptides, and the peptides were sampled onto a $100 \mu \mathrm{m} \times 10 \mathrm{~cm}$ fused silica emitter packed with reversed-phase ReproSil-Pur C18-AQ resin ( $3 \mu \mathrm{m}$ and $120 \AA \AA$; Ammerbuch). Linear gradients of $5-32 \%$ acetonitrile in $0.1 \%$ formic acid were used to elute the sample at a flow rate of $300 \mathrm{nl} / \mathrm{min}$ for $50 \mathrm{~min}$. An LTQ Orbitrap Elite mass spectrometer (ThermoFisher) equipped with a nanoelectrospray ion source (ProxeonBiosystems) was used to acquire the mass spectra data. Collision-induced dissociation (normalized collision energy, $35 \%$; activation Q, 0.250 ; activation time, $10 \mathrm{~ms}$ ) was used to perform fragmentation in the LTQ with a target value of 3000 ions. To search the raw files, the SEQUEST engine against a database from the Uniprot protein sequence database was used. Parameters were set as follows: protein modifications were set to carbamidomethylation (C) (fixed), oxidation (M) (variable), and phosphorylation ( $\mathrm{S}, \mathrm{T}, \mathrm{Y})$ (variable); the enzyme specificity was set to trypsin; a maximum missed cleavages were set to 2; the precursor ion mass tolerance was set to $10 \mathrm{ppm}$, and MS/MS tolerance was set to $0.5 \mathrm{Da}$.

Co-immunoprecipitation and immunoblot analysis. HEK293T cells were lysed by Co-IP lysis buffer containing $150 \mathrm{mM} \mathrm{NaCl}, 1 \mathrm{mM}$ EDTA, $20 \mathrm{mM}$ Tris- $\mathrm{HCl} \mathrm{pH}$ 8.0, 10\% (vol/vol) glycerol, $0.5 \%$ (vol/vol) NP40, and Protease inhibitor Cocktail (Roche). Cell lysates were subjected to SDS-PAGE and immunoblot analysis. For the co-immunoprecipitation assay, IgG or the appropriate antibodies were added into the cell lysates, followed by incubation with Protein A/G beads (EMD 
a

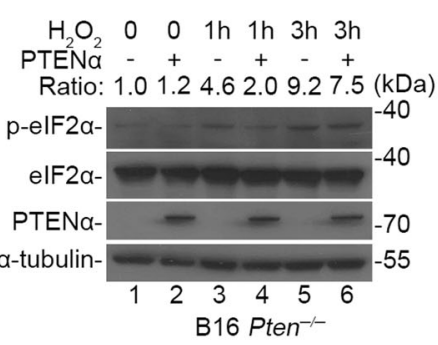

C

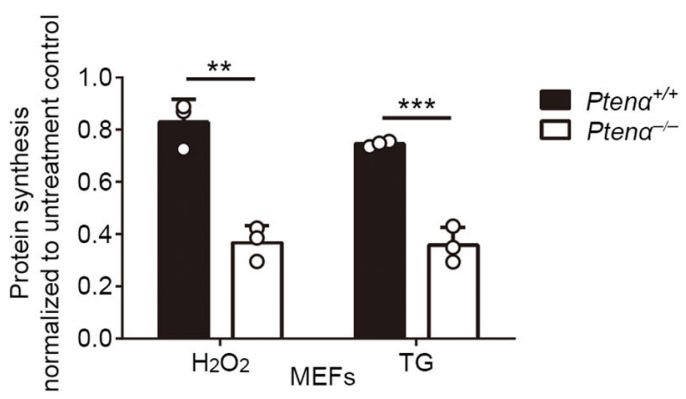

e

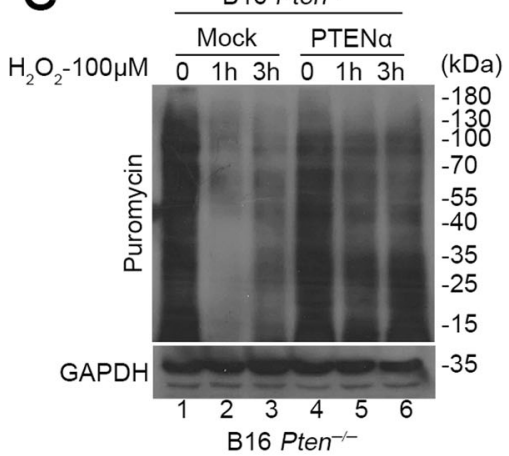

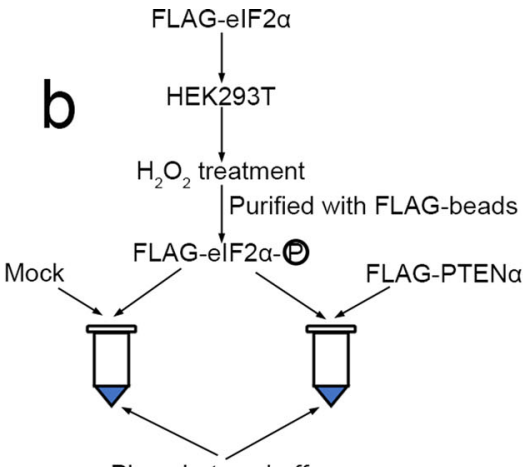

Phosphatase buffer

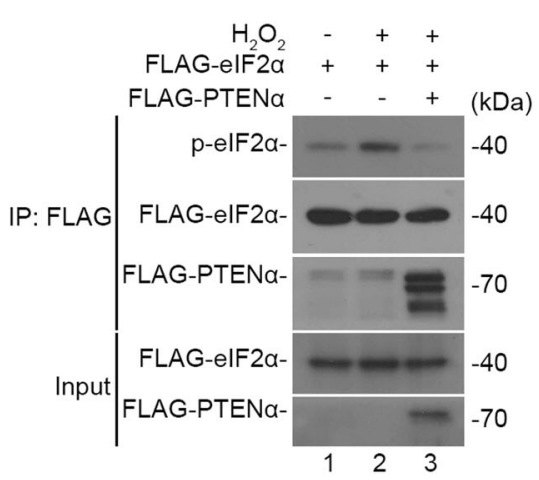

d

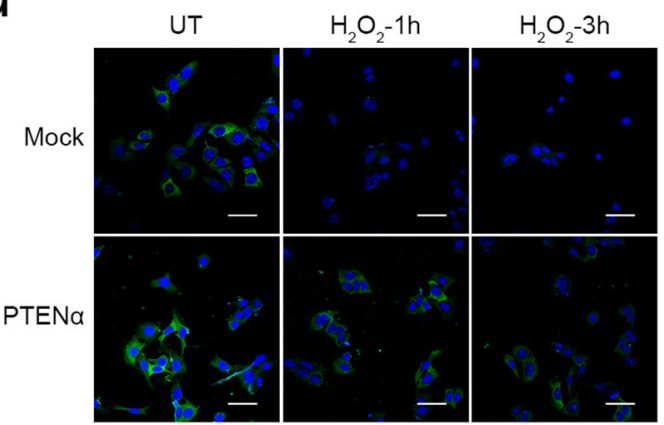

B16 Pten $^{-1}$ f

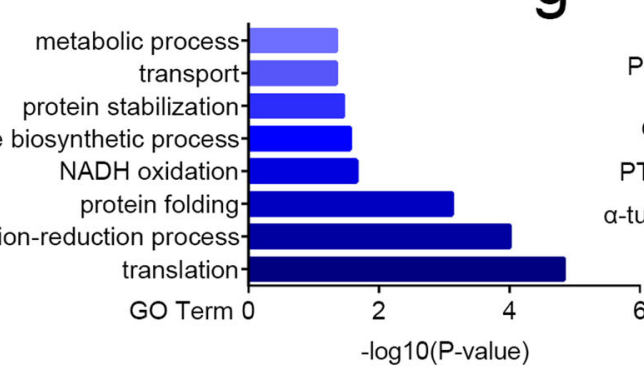

$\mathrm{HO} \quad \mathrm{O} 0 \mathrm{~h}$ h $1 \mathrm{~h} 3 \mathrm{~h} 3 \mathrm{~h}$ PTENa - + - + - + Ratio: $1.00 .90 .40 .70 .20 .5(\mathrm{kDa})$

L-proline bi

Gpx4 PTENa-

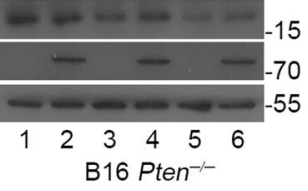

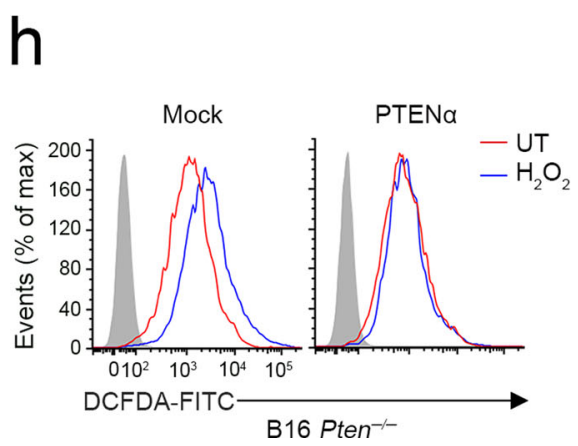

Millipore) for at least $4 \mathrm{~h}$. The precipitants were washed with PBS-N (PBS with $0.1 \%$ (vol/vol) NP40) four times, using for immune blotting analysis.

In vitro phosphatase assay. HEK293T cells were transfected to express FLAGtagged eIF2 $\alpha$ and treated with $100 \mu \mathrm{M} \mathrm{H}_{2} \mathrm{O}_{2}$ to induce phosphorylation of eIF2 $\alpha$. Then the phosphorylated eIF $2 \alpha$ was precipitated with FLAG beads, and incubated with precipitated FLAG-tagged PTENa in phosphatase buffer $(20 \mathrm{mM}$ Hepes $\mathrm{pH}=7.2,1 \mathrm{mM} \mathrm{DTT}, 1 \mathrm{mM} \mathrm{MgCl}$, and $1 \mathrm{mM}$ EDTA) for one hour. The proteins were eluted with FLAG peptide and subjected to immunoblot analysis.

Puromycin incorporation assay. Indicated cells were treated with $\mathrm{H}_{2} \mathrm{O}_{2}(100 \mu \mathrm{M})$ for indicated hours, followed by pulsing with puromycin $(50 \mu \mathrm{g} / \mathrm{mL})$ for $30 \mathrm{~min}$.

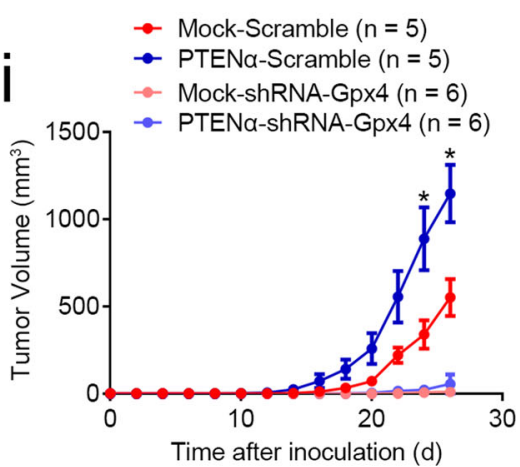


Fig. 7 PTEN $\alpha$ maintains protein synthesis of peroxidases. a Mock or PTEN $\alpha$ expressing Pten ${ }^{-/}-$B16 cells were treated with $100 \mu M \mathrm{H}_{2} \mathrm{O}_{2}$ for indicated hours, followed by immunoblot analysis with anti-elF2 $\alpha$ and anti-phospho-elF $2 \alpha$ antibodies. Gray values of total elF $2 \alpha$ and phosphorylated elF $2 \alpha$ were determined, and the relative ratio of phosphorylated elF $2 \alpha$ to total elF $2 \alpha$ was indicated. $\mathbf{b}$ In vitro phosphatase assay of FLAG-tagged PTEN $\alpha$ on phosphorylated elF2 $\alpha$. Phosphorylation level of elF2 $\alpha$ was assessed by immunoblot analysis with anti-phospho-elF2 $\alpha$ antibodies. c ${ }^{35}$ S-metabolic labeling analysis of Pten $\alpha^{+/+}$and $P$ ten $\alpha^{-/-}$MEFs treated with $100 \mu \mathrm{M} \mathrm{H}_{2} \mathrm{O}_{2}$ or $500 \mathrm{nM}$ TG for $3 \mathrm{~h}$. Gray values of autoradiographs were determined and used for statistical analysis (pooled samples, $n=3$ independent experiments, mean $\pm \mathrm{SD},{ }^{\star \star} P=0.0020,{ }^{\star \star \star} P=0.0007$ ). $\mathbf{d}$ and e Puromycin incorporation assay of Mock or PTEN $\alpha$ expressing Pten ${ }^{-/-}$B 16 cells treated with $100 \mu \mathrm{M} \mathrm{H}_{2} \mathrm{O}_{2}$ for indicated hours. Puromycin-labeled proteins were identified with confocal fluorescence microscopy (d) and immunoblot analysis (e). Scale bars, $100 \mu \mathrm{m}$. UT untreatment. $\mathbf{f}$ Pten $\alpha^{+/+}$and Pten $\alpha^{-/-}$MEFs were treated with $100 \mu \mathrm{M} \mathrm{H}_{2} \mathrm{O}_{2}$, followed by mass spectrometry analysis. Proteins that were significantly decreased in Pten $\alpha^{-/-}$MEFs were analyzed using DAVID with Gene Ontology (GO) terms. g Mock or PTEN $\alpha$ expressing Pten ${ }^{-/-}$B16 cells were treated with $100 \mu \mathrm{M} \mathrm{H}_{2} \mathrm{O}_{2}$ for indicated hours, and expression level of GPX4 was assessed by immunoblot analysis with anti-GPX4 antibody. Gray values of GPX4 and $\alpha$-tubulin were determined, and the relative ratio of GPX4 to $\alpha$-tubulin was indicated. $\mathbf{h}$ Flow cytometry analysis of Mock or PTEN $\alpha$ expressing Pten ${ }^{-/-}$B16 cells treated with $\mathrm{H}_{2} \mathrm{O}_{2}(100 \mu \mathrm{M})$ for indicated hours and stained with DCFDA. Mean fluorescence intensities of DCFDA were used for statistical analysis $(n=3$ cell cultures, mean $\left.\pm \mathrm{SD},{ }^{\star \star \star \star} P<0.0001\right)$. All live cells were gated. UT, untreatment. i Endogenous Gpx4 expression in B16-Pten ${ }^{-/-}$cells was knockdown using shRNA targeting Gpx4 or scramble shRNA. Then the cells were transfected to express Mock or PTEN $\alpha$ and subjected to cancer vaccination model. The tumor volumes were monitored over time (Scramble, $n=5$ mice; shRNA, $n=6$ mice, mean \pm s.e.m., ${ }^{\star} P(\mathrm{~d} 24)=0.0238,{ }^{\star} P(\mathrm{~d} 26)=0.0158$ ). Statistical significances between scramble groups were shown. Statistical significance was assessed by a two-tailed unpaired Student's $t$ test $(\mathbf{c}, \mathbf{h}, \mathbf{i})$. Data are representative of two (a, $\mathbf{b}, \mathbf{d}, \mathbf{e}, \mathbf{g}-\mathbf{i})$ independent experiments or pooled from three (c) independent experiments. Source data are provided as a Source Data file. See also Supplementary Fig. 10.

cancer vaccination model. The tumors were collected when the tumor volumes of Mock groups reached $200 \mathrm{~mm}^{3}$. The TILs were isolated, and $\mathrm{CD}_{4} 5^{+} 7 \mathrm{AAD}^{-}$cells were sorted, using for scRNA-seq with two replicates. Each sample is a mix of cells from two mice. Library construction and 10x scRNA-seq were performed by Berry Genomics. Sequencing was performed in different sequencing lanes in Illumina NovaSeq 6000.

For raw data processing and quality control, Cell Ranger (version 6.0.1) was used to produce a raw unique molecular identifier (UMI) count matrix through processes including cellular barcodes demultiplex, mapping reads to the transcriptome and generating quantitative matrix. The matrix was converted into a Seurat object by the $\mathrm{R}$ package Seurat (version 4.0.1). Low-quality cells with UMI numbers $>5000$ or $<300$, or have over 10\% mitochondrial-derived UMI counts were removed. 25,098 remained single cells were applied in downstream analyses.

Subsequently, the count matrix was normalized with the SCTransform function. Then we performed integration on the four datasets. In this process, potential Anchors were created with the FindIntegrationAnchors function using top 3000 variable genes, and IntegrateData function was used to integrate data and create a new matrix with 3000 features, in which potential batch effect was regressed out.

To reduce the dimensionality of the scRNA-Seq dataset, principal component analysis (PCA) was performed. Top 30 PCs were used to perform the downstream analysis with Elbowplot function. Subsequently, t-distributed stochastic neighbor embedding (tSNE) and uniform manifold approximation and projection (UMAP) were performed. The FindClusters function was used to divide all cells into 22 clusters with resolution set as 0.6 , and the FindAllmarkers function was performed to identify preferentially expressed genes in clusters. Based on these genes, the clusters were identified as 12 cell types, and visualized with $2 \mathrm{D}$ tSNE plots, and the heatmap plot was made with the DoHeatmap function. The proportion of each cell type in the four samples were counted by the R package dplyr (1.0.6), and the bar graph were drawn using R package ggplot2 (3.3.3).

For further analysis of the subpopulation of Cd8T cells, all Cd8T cells were divided into four clusters with the FindClusters function, with resolution set as 0.08 . The FindAllMarkers function was performed to identify preferentially expressed genes in the clusters. Based on these genes, four clusters were identified as four cell types, and visualized with 2D UMAP plot. Heatmap plot was made using the DoHeatmap function, and the proportion of each cell type in the Cd8T cells were counted by the R package dplyr (1.0.6). The bar graph was made using the R package ggplot2 (3.3.3). We also drawn the violin plots to show the expression of important genes in the Cd8T cells with the VlnPlot function.

Bioinformatics. Mutations, mRNA expression data and clinical data were from cBioportal $^{31,32}$. Stop gained mutations includes all the stop-gained and frameshift mutations early terminated after $\mathrm{H}^{185}$ (last amino acid of phosphatase tensin-type domain of PTEN). Loss of phosphatase activity mutations were from UniProt database.

For gene-set enrichment analysis (GSEA), differentially expressed genes or proteins were analyzed using applications from Broad Institute 21 (http:// www.broad.mit.edu/gsea/software/software_index.html) with default parameters. GO gene sets were downloaded from the 23 Broad Institute Molecular Signature Database (MSigDB)

Statistics and reproducibility. Prism GraphPad software v6.01 was used for analysis. The statistical significance between different groups was calculated with an unpaired Student's $t$-test or log-rank (Mantel-Cox) test. To compare three or more means, one or two-way ANOVA followed by Tukey's multiple comparisons test was used. $P<0.05$ was considered significant. All experiments were independently replicated at least two times and similar results were generated.
Reporting summary. Further information on research design is available in the Nature Research Reporting Summary linked to this article.

\section{Data availability}

The mass spectrometry proteome data generated in this study have been deposited in the Integrated Proteome Resources (iProX), an official member of ProteomeXchange Consortium, with the accession number IPX0002523000 and IPX0003269000. The scRNAseq data have been deposited in the GEO database with the accession code GSE178258. Sequencing data of PTEN in Molt4 and Jurkat cells have been deposited in the NCBI Genbank nucleotide database under accession code MZ615337, MZ615338 and MZ615339. Phosphatase-inactive PTEN mutations are from Uniprot database (https:// www.uniprot.org/uniprot/P60484). Clinical data of the tumor patients carrying PTEN mutations from the TCGA (UCEC, PanCancer Atlas) and Metastatic Colorectal Cancer databases (MSKCC, Cancer Cell 2018 ${ }^{33}$ ) can be acquired from cBioPortal (https:// www.cbioportal.org). GO gene-sets are from MSigDB (http://www.gsea-msigdb.org/gsea/ downloads.jsp). The remaining data are available within the Article, Supplementary Information or Source Data file. Source data are provided with this paper.

Received: 25 September 2020; Accepted: 4 August 2021; Published online: 26 August 2021

\section{References}

1. Lee, Y.-R., Chen, M. \& Pandolfi, P. P. The functions and regulation of the PTEN tumour suppressor: new modes and prospects. Nat. Rev. Mol. Cell Biol. 19, 547-562 (2018).

2. Song, M. S., Salmena, L. \& Pandolfi, P. P. The functions and regulation of the PTEN tumour suppressor. Nat. Rev. Mol. Cell Biol. 13, 283-296 (2012).

3. Hollander, M. C., Blumenthal, G. M. \& Dennis, P. A. PTEN loss in the continuum of common cancers, rare syndromes and mouse models. Nat. Rev. Cancer 11, 289-301 (2011).

4. Georgescu, M. M., Kirsch, K. H., Akagi, T., Shishido, T. \& Hanafusa, H. The tumor-suppressor activity of PTEN is regulated by its carboxyl-terminal region. Proc. Natl Acad. Sci. USA 96, 10182-10187 (1999).

5. Wang, $\mathrm{H}$. et al. Allele-specific tumor spectrum in pten knockin mice. Proc. Natl Acad. Sci. USA 107, 5142-5147 (2010).

6. Papa, A. et al. Cancer-associated PTEN mutants act in a dominant-negative manner to suppress PTEN protein function. Cell 157, 595-610 (2014).

7. Chen, D. S. \& Mellman, I. Elements of cancer immunity and the cancer-immune set point. Nature 541, 321-330 (2017).

8. Beatty, G. L. \& Gladney, W. L. Immune escape mechanisms as a guide for cancer immunotherapy. Clin. Cancer Res. 21, 687-692 (2015).

9. Kalbasi, A. \& Ribas, A. Tumour-intrinsic resistance to immune checkpoint blockade. Nat. Rev. Immunol. 20, 25-39 (2020).

10. Kim, J. M. \& Chen, D. S. Immune escape to PD-L1/PD-1 blockade: seven steps to success (or failure). Ann. Oncol. 27, 1492-1504 (2016).

11. Martinvalet, D., Dykxhoorn, D. M., Ferrini, R. \& Lieberman, J. Granzyme A cleaves a mitochondrial complex I protein to initiate caspase-independent cell death. Cell 133, 681-692 (2008).

12. Wang, W. et al. CD8+ T cells regulate tumour ferroptosis during cancer immunotherapy. Nature 569, 270-274 (2019). 
13. Zhou, Z. et al. Granzyme A from cytotoxic lymphocytes cleaves GSDMB to trigger pyroptosis in target cells. Science 368, eaaz7548 (2020).

14. Hopkins, B. D. et al. A secreted PTEN phosphatase that enters cells to alter signaling and survival. Science 341, 399-402 (2013).

15. Liang, H. et al. PTENa, a PTEN isoform translated through alternative initiation, regulates mitochondrial function and energy metabolism. Cell Metab. 19, 836-848 (2014).

16. Shen, S.-M. et al. PTEN $\alpha$ and PTEN $\beta$ promote carcinogenesis through WDR5 and H3K4 trimethylation. Nat. Cell Biol. 21, 1436-1448 (2019).

17. Ghandi, M. et al. Next-generation characterization of the Cancer Cell Line Encyclopedia. Nature 569, 503-508 (2019).

18. Wherry, E. J. \& Kurachi, M. Molecular and cellular insights into T cell exhaustion. Nat. Rev. Immunol. 15, 486-499 (2015).

19. Hangartner, L., Zinkernagel, R. M. \& Hengartner, H. Antiviral antibody responses: the two extremes of a wide spectrum. Nat. Rev. Immunol. 6 , 231-243 (2006).

20. Song, J. et al. The deubiquitinase OTUD1 enhances iron transport and potentiates host antitumor immunity. EMBO Rep. 22, e51162 (2021).

21. McCormick, C. \& Khaperskyy, D. A. Translation inhibition and stress granules in the antiviral immune response. Nat. Rev. Immunol. 17, 647-660 (2017).

22. Buchan, J. R. \& Parker, R. Eukaryotic stress granules: the ins and outs of translation. Mol. Cell 36, 932-941 (2009).

23. Khong, A. et al. The stress granule transcriptome reveals principles of mRNA accumulation in stress granules. Mol. Cell 68, 808-820.e805 (2017).

24. Zid, B. \& O'Shea, E. Promoter sequences direct cytoplasmic localization and translation of mRNAs during starvation in yeast. Nature 514, 117-21 (2014).

25. Peng, W. et al. Loss of PTEN promotes resistance to $\mathrm{T}$ cell-mediated immunotherapy. Cancer Discov. 6, 202-216 (2016).

26. Okkenhaug, K., Graupera, M. \& Vanhaesebroeck, B. Targeting PI3K in cancer: impact on tumor cells, their protective stroma, angiogenesis, and immunotherapy. Cancer Discov. 6, 1090-1105 (2016).

27. Riquelme, S. A. et al. Cystic fibrosis transmembrane conductance regulator attaches tumor suppressor PTEN to the membrane and promotes anti Pseudomonas aeruginosa. Immun. Immun. 47, 1169-1181 (2017).

28. Mao, H., Zhao, Y., Li, H. \& Lei, L. Ferroptosis as an emerging target in inflammatory diseases. Prog. Biophys. Mol. Biol. 155, 20-28 (2020).

29. Ubellacker, J. M. et al. Lymph protects metastasizing melanoma cells from ferroptosis. Nature 585, 113-118 (2020).

30. Wang, P. et al. PTENa modulates CaMKII signaling and controls contextual fear memory and spatial learning. Cell Rep. 19, 2627-2641 (2017).

31. Cerami, E. et al. The cBio cancer genomics portal: an open platform for exploring multidimensional cancer genomics data. Cancer Discov. 2, 401 (2012).

32. Gao, J. et al. Integrative analysis of complex cancer genomics and clinical profiles using the cBioPortal. Sci. Signal. 6, pl1-pll (2013).

33. Yaeger, R. et al. Clinical sequencing defines the genomic landscape of metastatic colorectal cancer. Cancer Cell 33, 125-136.e123 (2018).

\section{Acknowledgements}

This work was supported by grants including the National Key Research and Development Program of China (Grant 2016YFA0500302 to Y.Y.), the National Natural Science Foundation of China (Key grants 82030081 and 81874235 to Y.Y., and grant 82022032 and 81991505 to D.L.), the Lam Chung Nin Foundation for Systems Biomedicine, Clinical Medicine Plus X-Young Scholars Project, Peking University, the fundamental research funds for the Central Universities (No. PKU2021LCXQ026 to D.L.), and the Fundamental Research Funds for the Central Universities (No. BMU2018YJ003 to D.L).

\section{Author contributions}

Y.S., D.L. and Yuxin Y. conceived the study and designed experiments. Y.S. and D.L. performed the most of experiments and analyzed the data. J.S., W.H., Yue Y. and F.Q. assisted in some experiments. Y.J. provided technical assistance. Xuyang Z. performed mass spectrometry analysis. Xin Z. and G.Z. analyzed the sc-RNAseq data. L.L., Z.L., Y.J. Y.L. and H.L. provided reagents. D.L. and Yuxin Y. supervised the research. Y.S., D.L. and Yuxin Y. wrote the paper.

\section{Competing interests}

The authors declare no competing interests.

\section{Additional information}

Supplementary information The online version contains supplementary material available at https://doi.org/10.1038/s41467-021-25417-6.

Correspondence and requests for materials should be addressed to D.L. or Y.Y.

Peer review information Nature Communications thanks Weiyi Peng and the other, anonymous, reviewer(s) for their contribution to the peer review of this work. Peer reviewer reports are available.

Reprints and permission information is available at http://www.nature.com/reprints

Publisher's note Springer Nature remains neutral with regard to jurisdictional claims in published maps and institutional affiliations.

\begin{abstract}
(c) (i) Open Access This article is licensed under a Creative Commons By Attribution 4.0 International License, which permits use, sharing adaptation, distribution and reproduction in any medium or format, as long as you give appropriate credit to the original author(s) and the source, provide a link to the Creative Commons license, and indicate if changes were made. The images or other third party material in this article are included in the article's Creative Commons license, unless indicated otherwise in a credit line to the material. If material is not included in the article's Creative Commons license and your intended use is not permitted by statutory regulation or exceeds the permitted use, you will need to obtain permission directly from the copyright holder. To view a copy of this license, visit http://creativecommons.org/ licenses/by/4.0/.
\end{abstract}

(c) The Author(s) 2021 